\title{
8. ORIGIN OF THE LATE CENOZOIC SEDIMENTS OF THE ICELANDIC BASIN, DSDP SITE 348, LEG 38
}

\author{
M.P. Nesterova, F.A. Scherbakov, A.Ja. Shevchenko, N.W. Turanskaja, W.P. Kazakova, \\ A.G. Samosudova, T.G. Kuzmina, and A.N. Rudakova, Analytical Laboratory, \\ P.P. Shirshov Institute of Oceanology, Academy of Sciences, USSR
}

\section{INTRODUCTION}

Site 348 attracted a great deal of interest because the hole was drilled within the Icelandic Basin, which represents a large depression in the Icelandic Plateau.

Laboratory studies consisted of a determination of: sediment grain sizes, the mineralogic composition of the coarse silt fraction ( 0.1 to $0.05 \mathrm{~mm}$ ), and the chemical composition and the composition of clay minerals in the less than $0.001 \mathrm{~mm}$ size fraction.

\section{Data Presentation}

The data obtained are listed in Tables 1-9. The data in Table 1 are average values that characterize the sediments and rocks of each of the three stratigraphic units by the shipboard party (see Site Report, Chapter 8 , this volume). The tables also present data on the terrigenous, biogenic, volcanogenic, and authigenic sediment components. The data on chemical composition (Table 2) indicate the content of biogenic silica and $\mathrm{CaCO}_{3}$ in the sediment. The iron content $\left(\mathrm{Fe}_{2} \mathrm{O}_{3}\right)$ (Table 4) indicates the presence of both terrigenous and authigenic material. Components in the separate grain size fractions are grouped in such a manner so as to characterize most accurately the various components of the sediment. Table 3 also includes data on the quantity of particles with a size of less than $5 \mu \mathrm{m}$. It was assumed that these particles consist almost entirely of clay minerals.

The data presented on the content of separate minerals in the 0.1-0.05 mm fraction (Table 1) concentrate on a group of the most typical terrigenous minerals: quartz, potassium feldspars, hornblende, and weathered grains (chiefly rock debris, plagioclase feldspars). For the volcanogenic sediments, the data show the contents of varieties such as glass and various types of more or less altered particles of volcanic ash. The authigenic group included pyrite and marcasite, iron hydroxides, glauconite, and zeolites which have been formed in the process of devitrification and palagonitization of the primary volcanogenic ash material. However, some zeolites probably have a volcanic origin.

From analysis of the data, it is apparent that each of the three lithologic units distinguished differ both in composition and genesis. However, the interpretations on the composition and genesis of these sediments are somewhat different from those of the shipboard party (see Site Report, Chapter 8, this volume).

\section{DISCUSSION}

Based on the data available, it is concluded that the sediments of all three units in Hole 348 are essentially clay sediments. The average percentage of the clay content calculated for the three units is over $70 \%$ (Table 3 ). The major bulk of these particles is represented by clay minerals, and a considerable portion of this clay material is authigenic. The material, which has been altered and converted into these clays, was probably of a volcanogenic origin and not terrigenous (see however, Site Report, Chapter 8, this volume).

\section{Biogenic Components (Carbonate and Silica)}

Biogenic calcium carbonate has an extremely minor content in the Cenozoic sediments. Rarely, except in separate interlayers, does the $\mathrm{CaCO}_{3}$ content reach $15 \%$. However, biogenic amorphous silica is far more important, although the $\mathrm{SiO}_{2}$ content in the samples studied hardly exceeds $8 \%$ (Table 2). A study of smear slides and thin sections of these sediments under the scanning electron microscope shows a high content of siliceous skeletons. It is believed that although the skeletal volume may be high, the mass contained in the skeletons is low. On the other hand, the mass of clay material in a similar volume is appreciably higher.

\section{Volcanic Components}

For the volcanic constituents, the predominant mass has been transported as ash particles to oceanic regions. The sediment contains particles of volcanic glass whose size corresponds chiefly to silt and (partially) coarse clay fractions.

Volcanic glass, under certain conditions, will comprise the major portion of such fractions; however, the main mass of volcanic glass appears to have been completely altered and converted to clay.

\section{Studies of Volcanic Components}

Volcanic material in the coarse fraction consists of heavy (specific weight $2.9 \mathrm{~g} / \mathrm{cc}$ ) and light (specific weight less than $2.9 \mathrm{~g} / \mathrm{cc}$ ) subfractions. The material includes volcanic glass that contained both the basic $(N>1.54$ [dominant type]) and acid varieties $(N<1.54)$. Frequently, a significant portion of the volcanic material was represented by devitrified and more or less altered glass, particularly the palagonitized variety. These can be considered varieties of ash particles.

Also detected in the volcanogenic component of the coarse silt (heavy subfraction) were monoclinic pyrox- 
TABLE

Composition of Sediments Cored at Site 348

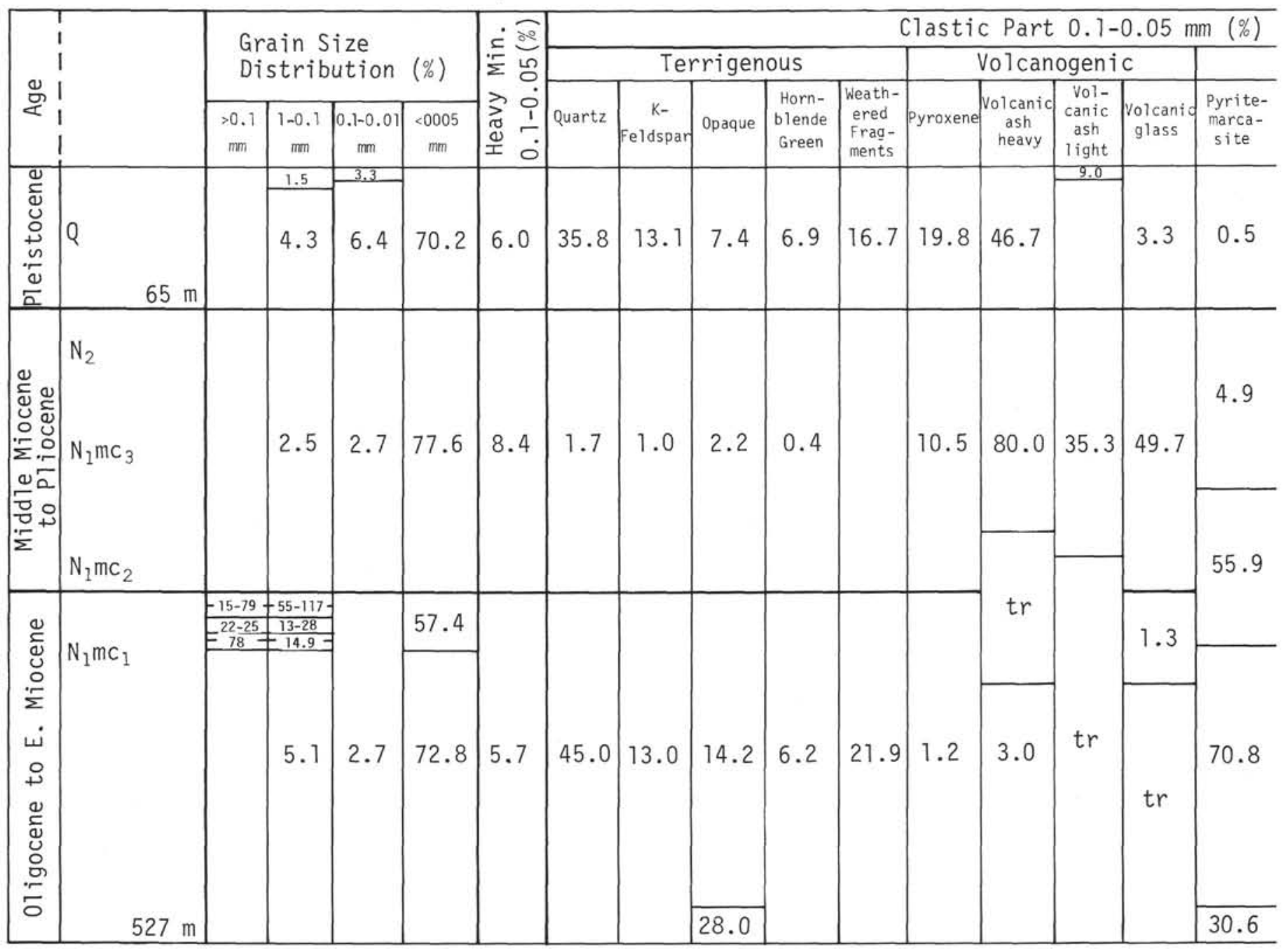

enes (augite). However, some of the pyroxenes may have been transported (especially in Pleistocene times) as terrigenous components from Iceland.

\section{Authigenic Components}

Authigenic minerals formed by diagenesis include pyrite and marcasite (in the heavy subfraction of the coarse silt fraction). These are grouped in Table 1 as iron sulfides $\left(\mathrm{FeS}_{2}\right)$ and include iron hydroxides in the form of limonite and glauconite. These varieties, together with conspicuous zeolites, are commonly present in the thin bedded clay fractions. The zeolites were observed in the diffraction (X-ray) patterns of sediments of the less than $1 \mu \mathrm{m}$ fraction, however, it is believed they are accessory minerals.

\section{Clay Minerals}

Important in the definition of sediment composition and genesis is determining the composition of clay minerals. The study utilized the data from X-ray diffraction and SEM microphotography. Detected were montmorillonite and hydromica, as well as chlorite, zeolites, and mixed-layer clays of varying compositions.
Calculations of the quality of these minerals in the less than $1 \mu \mathrm{m}$ fraction are based on the technique of Brindley (1965).

Montmorillonite is among the clays which comprise a major portion of the section. Moreover, montmorillonite, in pre-Pleistocene deposits, predominates and constitutes, locally, up to $100 \%$ in the less than $1 \mu \mathrm{m}$ fraction. It is believed that the main portion of this montmorillonite is authigenic, formed as a result of transformation of basic volcanic glass.

Thus, analysis of the clay fraction of the sediment seems to provide substantial grounds that the major portion of the pre-Pleistocene sediments in the Icelandic Basin is primarily volcanogenic. These volcanogenic components have been subsequently transformed in varying degrees into authigenic components.

\section{GENERAL CONCLUSIONS}

Considerations of the data from the lithologic studies of the core samples of Hole 348 permit some general conclusions on the composition and genesis of the sedimentary material. 
TABLE 1 - Continued

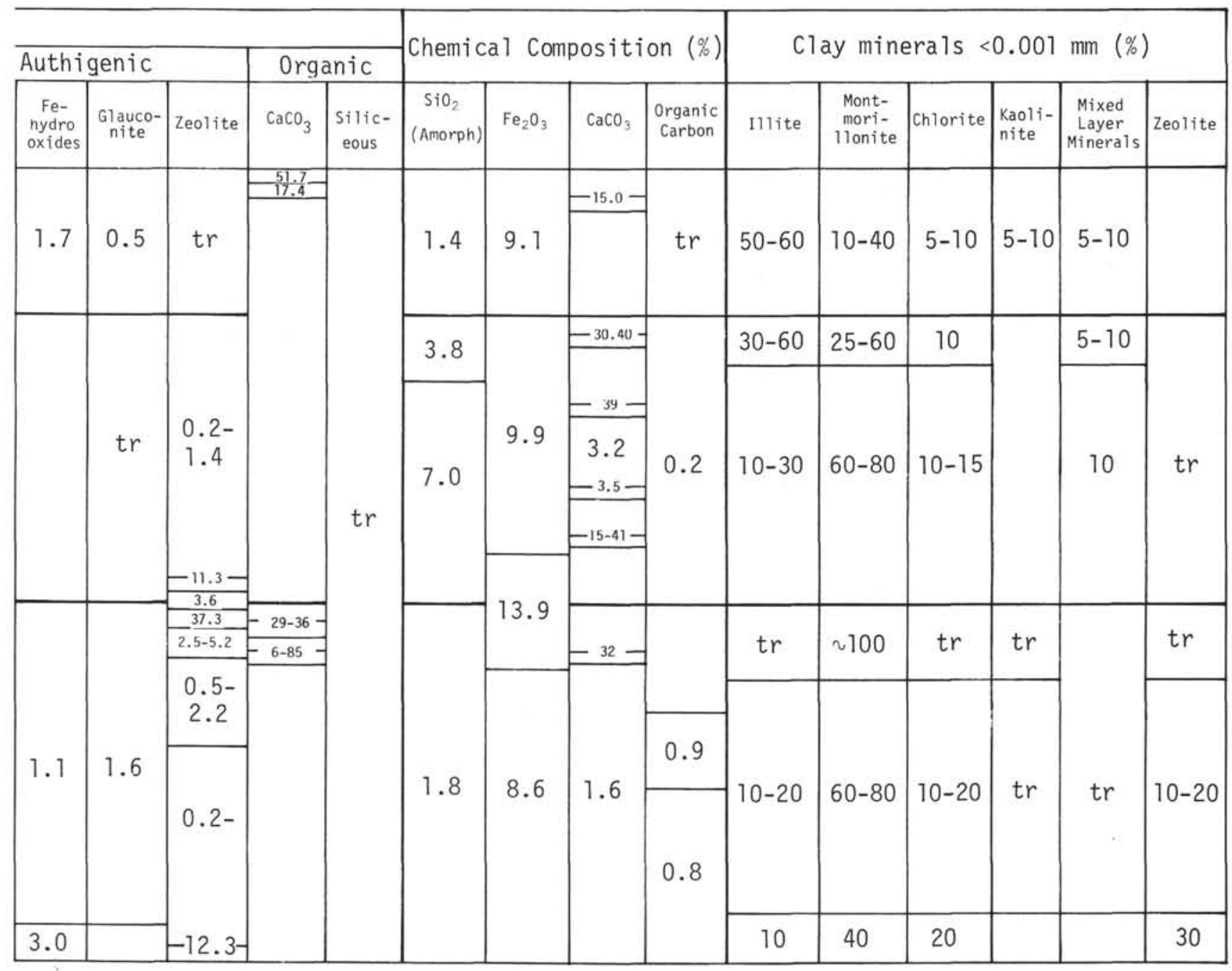

Despite the homogeneous composition of clay in the sedimentary section, there are very minor variations in the composition and content of accessory terrigenous, biogenic, volcanogenic, authigenic, and clay components. These variations support the division of the sediments into three stratigraphic units. There is also a general agreement of lithologic and stratigraphic boundaries.

\section{Unit 3}

The "accessory" components in the silt portion of the sediment section are almost exclusively "terrigenous" in the lower portion of Unit 3 (Oligocene and early Miocene). Volcanogenic constituents are practically absent in the silt fraction, however, there is a great deal of quartz, weathered plagioclase, or other minerals. The silt is also characterized by the highest content of heavy ore minerals (Table 1). Noteworthy is the low content of biogenic $\left(\mathrm{SiO}_{2}, \mathrm{CaCO}_{3}\right)$ components. Interestingly, however, the sediments do have a high $\mathrm{C}_{\text {orR }}$ content.

A direct correlation seems to exist with $\mathrm{C}_{\text {org }}$ and the percentage of authigenic pyrite and marcasite in Unit 3. Less common is glauconite. In all probability, the high
$\mathrm{C}_{\text {org }}$ content present is the main factor that led to the origin (via diagenesis) of these authigenic components. Also of interest is the fact that all these deposits are the oldest of those cored.

Although all sediment sections are characterized by a generally low zeolite content in the silty sediments, Unit 3 has a high content (Table 1). The distribution of these zeolites in the coarse silts shows a tendency to increase in content from the top. An exception is the lowermost portion of Unit 3, where large zeolite crystals are abundant (Figures 1 and 2).

The clay component in Unit 3 has a quite different character. The less than $1 \mu \mathrm{m}$ fraction is completely composed of montmorillonite, especially at the top of the unit (early-Miocene). X-ray diffraction fails to reveal but a trace of other minerals. An exception is the lowermost beds (late Oligocene) above basalts. Here, apart from the predominant montmorillonite, there is a considerable amount of chlorite, hydromica, and kaolinite. Zeolite is also conspicuous. The composition of these clays provides grounds to indicate that this clay has formed from ash material and basaltic lavas. The absence of the ash in larger size fractions indicates an 
TABLE 2A

Results of Immersion Mineralogical Analysis of the 0.1-0.5 mm Fraction Samples (Heavy Minerals) From Hole 348 DSDP Leg 38

\begin{tabular}{|c|c|c|c|c|c|c|c|c|c|c|c|c|c|c|c|c|c|c|}
\hline $\begin{array}{c}\text { Sample } \\
\text { (Interval in } \mathrm{cm} \text { ) }\end{array}$ & 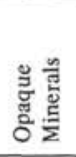 & 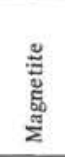 & 莺 & 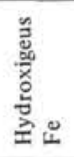 & 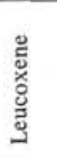 & 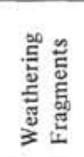 & 芯 & 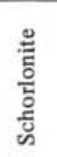 & 总 & 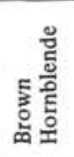 & 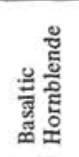 & 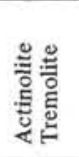 & 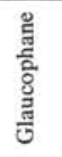 & 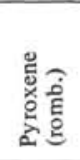 & 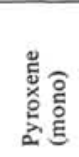 & 壱 & 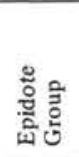 & 䓂 \\
\hline $1-1,70-72$ & 26 & 9 & 8 & 4 & & 3 & 4 & & 23 & & & & & 2 & 12 & & 4 & \\
\hline $1-1,70-72$ & 6.6 & 2.4 & 2.1 & 10 & & 1.6 & 1.0 & & 6.1 & & & & & 0.5 & 3.1 & & 1.0 & \\
\hline $1-3,100-104$ & 77 & 8 & 5 & 40 & 6 & 28 & 40 & & 49 & & & 5 & 1 & 6 & 34 & & 58 & 2 \\
\hline $1-3,100-104$ & 19.1 & 2.0 & 1.2 & 10.0 & 1.5 & 7.0 & 10.0 & & 12.3 & & & 1.2 & 0.2 & 1.5 & 8.5 & & 14.3 & 0.5 \\
\hline $1-4,100-102$ & & 5 & & 2 & & & 1 & & 6 & 3 & & 2 & & 4 & 37 & & 8 & 1 \\
\hline $1-4,100-102$ & & 0.7 & & 0.3 & & & 0.1 & & 0.8 & 0.4 & & 0.3 & & 0.5 & 5.1 & & 1.1 & 0.1 \\
\hline $1-5,90-92$ & 12 & 30 & 1 & 4 & 4 & 20 & 7 & & 21 & 18 & 5 & 2 & & 1 & 94 & 1 & 61 & \\
\hline $1-5,90-92$ & 3.5 & 8.0 & 0.3 & 1.1 & 1.1 & 5.4 & 1.9 & & 5.7 & 4.8 & 1.3 & 0.5 & & 0.3 & 25.3 & 0.3 & 16.4 & \\
\hline $1, \mathrm{CC}$ & 53 & 22 & & 24 & 4 & & 11 & & 61 & & 2 & 2 & & 18 & 68 & & 51 & \\
\hline $1, \mathrm{CC}$ & 13.9 & 5.8 & & 6.3 & 1.0 & & 2.9 & & 16.3 & & 0.5 & 0.5 & & 4.7 & 17.8 & & 13.4 & \\
\hline $2-1,98-100$ & & 35 & & 5 & & & 1 & & 34 & 11 & & 6 & & 4 & 72 & & 42 & \\
\hline $2-1,98-100$ & & 9.4 & & 1.3 & & & 0.2 & & 9.2 & 3.0 & & 1.6 & & 1.1 & 19.3 & & 11.3 & \\
\hline $2-2,50-52$ & & 29 & & 11 & & & 2 & & 17 & 5 & 1 & 1 & & 1 & 64 & 2 & 23 & 2 \\
\hline $2-2,50-52$ & & 6.8 & & 2.6 & & & 0.5 & & 4.0 & 1.1 & 0.2 & 0.2 & & 0.2 & 15.0 & 0.5 & 5.3 & 0.5 \\
\hline $2-4,99-101$ & 14 & & & 5 & 1 & 3 & 1 & & 9 & & & & & & 14 & 1 & 20 & \\
\hline $2-4,99-101$ & $49^{-}$ & & & - & - & - & - & & - & & & & & & - & - & - & \\
\hline $2, \mathrm{CC}$ & 49 & 19 & & 37 & 5 & 44 & 37 & & 89 & & 2 & 3 & & 16 & 80 & 1 & 61 & 1 \\
\hline $2, \mathrm{CC}$ & 10.2 & 3.9 & & 7.7 & 1.0 & 9.1 & 7.7 & & 18.2 & & 0.4 & 0.6 & & 33 & 16.5 & 0.2 & 12.6 & 0.2 \\
\hline $3-1,99-101$ & 53 & 6 & 1 & 12 & 5 & 31 & 13 & & 13 & & 2 & 2 & & 1 & 48 & & 18 & \\
\hline $3-1,99-101$ & 24.4 & 2.8 & 0.5 & 5.5 & 2.3 & 14.3 & 6.0 & & 6.0 & & 0.9 & 0.9 & & 0.5 & 21.0 & & 8.3 & \\
\hline $3-3,99-101$ & 33 & 45 & & 13 & 5 & 19 & 18 & & 68 & & 5 & 6 & & 5 & 77 & & 33 & 1 \\
\hline $3-3,99-101$ & 98 & 13.2 & & 3.8 & 1.5 & 5.6 & 5.3 & & 20.0 & & 1.5 & 1.2 & & 1.5 & 22.8 & & 9.8 & 0.3 \\
\hline $3-4,99-101$ & 78 & 15 & & 15 & 3 & 22 & 35 & 2 & 87 & & 1 & 4 & & 9 & 45 & 1 & 71 & \\
\hline $3-4,99-101$ & 18.4 & 3.5 & & 3.5 & 0.7 & 5.2 & 8.2 & 0.5 & 20.6 & & 0.2 & 0.9 & & 2.1 & 10.6 & 0.2 & 16.7 & \\
\hline $3-5,89-91$ & 43 & 14 & & 6 & & 18 & 7 & & 69 & & & 7 & & 8 & 94 & & 36 & \\
\hline $3-5,89-91$ & 13.5 & 4.4 & & 1.9 & & 5.7 & 2.2 & & 21.8 & & & 2.2 & & 2.5 & 29.7 & & 11.4 & \\
\hline $3, \mathrm{CC}$ & 38 & 34 & & 9 & & 141 & 10 & & 71 & & 1 & 9 & & 10 & 52 & & 44 & \\
\hline $3, \mathrm{CC}$ & 87 & 78 & & 2.0 & & 32.1 & 2.3 & & 16.2 & & 0.2 & 2.0 & & 23 & 11.8 & & 10.0 & \\
\hline $4-1,119-121$ & 17 & 30 & 3 & 13 & & & 7 & & 37 & 17 & & 3 & & 4 & 95 & & 12 & 2 \\
\hline $4-1,119-121$ & 4.5 & 8.2 & 0.8 & 35 & & & 1.9 & & 10.3 & 4.6 & & 0.8 & & 1.1 & 25.8 & & 3.3 & 0.5 \\
\hline $4-2,99-104$ & 5 & 10 & & 7 & & & 4 & & 31 & 1 & & 2 & & 3 & 123 & & 14 & \\
\hline $4-2,99-104$ & 1.1 & 2.2 & & 1.6 & & & 0.9 & & 7.1 & 0.2 & & 0.4 & & 0.7 & 27.6 & & 3.1 & \\
\hline $5-1,99-100$ & 6 & 6 & & 8 & & & 1 & & 3 & & & & & 1 & 11 & & 3 & \\
\hline $5-1,99-100$ & 1.3 & 1.3 & & 1.7 & & & 0.2 & & 0.6 & & & & & 0.2 & 2.3 & & 0.6 & \\
\hline $5-2,139-141$ & 2 & 10 & 9 & 7 & & 5 & 5 & & 29 & & & 3 & & 1 & 6 & 1 & 15 & \\
\hline $5-2,193,141$ & 0.4 & 1.9 & 17 & 1.3 & & 1.0 & 1.0 & & 5.6 & & & 0.6 & & 0.2 & 1.1 & 0.2 & 2.9 & \\
\hline $5, \mathrm{CC}$ & 12 & 43 & 9 & 91 & & & 1 & & 13 & & & & & & 20 & & 13 & \\
\hline $5, \mathrm{CC}$ & 2.8 & 10.1 & 2.1 & 21.2 & & & 0.2 & & 3.0 & & & & & & 4.7 & & 3.0 & \\
\hline $6-1,139-141$ & 12 & 4 & & & & & & & 7 & 3 & & & & 1 & 26 & & 5 & \\
\hline $6-1,139-141$ & 2.6 & 0.9 & & & & & & & 1.5 & 0.7 & & & & 0.2 & 5.7 & & 1.1 & \\
\hline $6-2,29-31$ & 21 & 7 & & 7 & & & & & 3 & & & & & 1 & 16 & & 5 & \\
\hline $6-2,29-31$ & 4.4 & 1.5 & & 1.5 & & & & & 0.6 & & & & & 0.2 & 3.4 & & 1.0 & \\
\hline $6-4,84-86$ & 19 & 5 & 17 & 6 & & 10 & & & 2 & & & & & & 22 & & 6 & \\
\hline $6-4,84-86$ & 4.0 & 1.1 & 3.6 & 1.3 & & 2.1 & & & 0.4 & & & & & & 4.7 & & 1.3 & \\
\hline $6-5,73-75$ & 12 & & 1 & 11 & & 7 & 4 & & 2.4 & & & 1 & & 1 & 29 & & .3 & \\
\hline $6-5,73-75$ & 2.3 & & 0.2 & 2.1 & & 1.3 & 0.8 & & 4.6 & & & 0.2 & & 0.2 & 5.5 & & 0.6 & \\
\hline $6, \mathrm{CC}$ & 10 & & & 4 & & & 1 & & 2 & & & & & 2 & 7 & & 2 & \\
\hline $6, \mathrm{CC}$ & 2.0 & & & 0.8 & & & 0.2 & & 0.4 & & & & & 0.4 & 1.6 & & 0.4 & \\
\hline $7-1,74-76$ & & 14 & 2 & 4 & & & 1 & & 2 & & & 2 & & & 37 & & 6 & \\
\hline $7-1,74-76$ & & 2.9 & 0.4 & 0.8 & & & 0.2 & & 0.4 & & & 0.4 & & & 7.7 & & 1.2 & \\
\hline $7-3,74-76$ & & 6 & 3 & 4 & & & & & 2 & & & & & & 15 & & & \\
\hline $7-3,74-76$ & & 1.4 & 0.7 & 0.9 & & & & & 0.4 & & & & & & 3.4 & & & \\
\hline $7-4,75-77$ & & 6 & 1 & 11 & & & & & 1 & & & & & & 12 & & 1 & \\
\hline $7-4,75-77$ & & 1.5 & 0.2 & 2.8 & & & & & 0.2 & & & & & & 31 & & 0.2 & \\
\hline $7, \mathrm{CC}$ & 11 & 5 & 21 & 3 & & & & & 2 & & & & & & 30 & & 6 & \\
\hline $7, \mathrm{CC}$ & 2.5 & 1.1 & 4.8 & 0.7 & & & & & 0.5 & & & & & & 6.8 & & 1.4 & \\
\hline $8-2,75-77$ & & 30 & 22 & 13 & & & 1 & & 1 & & & 1 & & & 157 & & 1 & \\
\hline $8-2,75-77$ & & 6.3 & 4.7 & 2.8 & & & 0.2 & & 0.2 & & & 0.2 & & & 34.0 & & 0.2 & \\
\hline $8-3,75-77$ & & 13 & 23 & 4 & & & & & & & & & & & 35 & & 2 & \\
\hline $8-3,75-77$ & & 3.2 & 5.7 & 1.0 & & & & & & & & & & & 8.6 & & 0.5 & \\
\hline $9-1,139-140$ & & 14 & & 5 & & & & & 1 & 1 & & & & & 40 & & 2 & \\
\hline $9-1,139-140$ & & 2.6 & & 0.9 & & & & & 0.2 & 0.2 & & & & & 7.3 & & 0.4 & \\
\hline $9-2,75-77$ & & 5 & 2 & 4 & & & & & & & & & & & 35 & & 4 & \\
\hline $9-2,75-77$ & & 0.9 & 0.4 & 0.8 & & & & & & & & & & & 6.5 & & 0.8 & \\
\hline $9-5,75-77$ & & 7 & 1 & 5 & & & & & 4 & & & & & & 36 & & & \\
\hline $9-5,75-77$ & & 1.3 & 0.2 & 0.9 & & & & & 0.7 & & & & & & 6.7 & & & \\
\hline $9, \mathrm{CC}$ & 19.0 & 4 & & 22 & & & & & & & & & & & 107 & & & \\
\hline $9, \mathrm{CC}$ & 40 & 0.9 & & 4.7 & & & & & & & & & & & 22.9 & & & \\
\hline $11-1,80-82$ & & 13 & 19 & 3 & & & & & & & & & & & 36 & & & \\
\hline $11-1,80-82$ & & 2.7 & 4.0 & 0.6 & & & & & & & & & & & 7.6 & & & \\
\hline $11-2,80-82$ & & 6 & 9.0 & $\begin{array}{l}0.0 \\
6\end{array}$ & & & & & & & & & & & 18 & & 1 & \\
\hline $11-2,80-82$ & & 1.4 & 2.1 & 1.4 & & & & & & & & & & & 4.2 & & 0.2 & \\
\hline $11-4,70-72$ & & 20 & 5 & 6 & & & & & & & & & & 1 & 75 & & & \\
\hline $11-4,70-72$ & & 3.8 & 0.9 & 1.1 & & & & & & & & & & 0.2 & 14.4 & & & \\
\hline $12-2,60-62$ & & 4 & 144 & & & & & & & 1 & & & & & 75 & & & \\
\hline $12-2,60-62$ & & 0.9 & 31.3 & & & & & & & 0.2 & & & & & 16.3 & & & \\
\hline $12-4,20-22$ & & 6 & 40 & & & & & & & & & & & 1 & 39 & & & \\
\hline $12-4,20-22$ & & 1.3 & 8.5 & & & & & & & & & & & 0.2 & 8.3 & & & \\
\hline $13-1,80-82$ & 6 & 4 & & 9 & & & & & & & & & & 1 & 26 & & 4 & \\
\hline
\end{tabular}


TABLE 2A - Continued

\begin{tabular}{|c|c|c|c|c|c|c|c|c|c|c|c|c|c|c|c|c|c|c|c|c|c|c|c|}
\hline 竞 & $\frac{0}{0}$ & 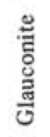 & ڤ્छ & 駦 & 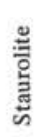 & 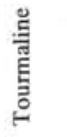 & 总 & 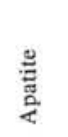 & $\stackrel{\mathscr{Z}}{\beth}$ & 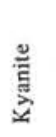 & 吾 & 预 & $\begin{array}{l}\bar{\Xi} \\
\text { है. } \\
\text { 己े }\end{array}$ & & 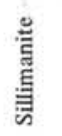 & 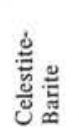 & 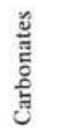 & 氖 & 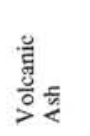 & 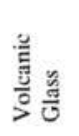 & 莙 & 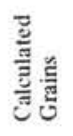 & 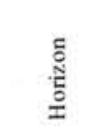 \\
\hline 1 & & & & & & 1 & 3 & 1 & & 2 & & & & & & & & & 274 & & & 380 & $70-72$ \\
\hline \multirow[t]{3}{*}{0.3} & & & & & & 0.3 & 0.8 & 0.3 & & 0.5 & & & & & & & & & 72.4 & & & & \\
\hline & & & & 8 & & 2 & 13 & 16 & 3 & & & & & & & & 1 & & & & & 402 & $100-104$ \\
\hline & & & & 2.0 & & 0.5 & 3.2 & 4.0 & 0.7 & & & & & & & & 0.2 & & & & & & \\
\hline 2 & & & & 1 & & & 2 & 2 & & & & & & & & & & & 651 & & & 727 & $100-102$ \\
\hline 0.3 & & & & 0.1 & & & 0.3 & 0.3 & & & & & & & & & & & 89.6 & & & & \\
\hline 1 & 2 & & & 8 & & 2 & 2 & 4 & & 3 & & & & 3 & 1 & 2 & & & 61 & & & 372 & $90-92$ \\
\hline 0.3 & 0.5 & & & 2.1 & & 0.5 & 0.5 & 1.1 & & 0.8 & & & & 0.8 & 0.3 & 0.5 & & & 16.3 & & & & \\
\hline 7 & 1 & & & 5 & & & 2 & 5 & 2 & & & 3 & & & & & & & 40 & & & 381 & \\
\hline 18 & 0.3 & & & 1.4 & & & 0.5 & 1.4 & 0.5 & & & 0.8 & & & & & & & 10.5 & & & & \\
\hline & & & & 4 & & 1 & 2 & 3 & & 2 & & & & & & & & & 149 & & & 372 & $98-100$ \\
\hline & 1 & & & 1.1 & & 0.2 & 0.5 & 0.8 & & & & 0.5 & & & & & & & 40.3 & & & & \\
\hline 0.5 & $\begin{array}{l}1 \\
0.2\end{array}$ & & & 5 & & 1 & 1 & & 1 & 2 & & 1 & & 2 & & & 2 & 1 & 250 & & & 429 & $50-52$ \\
\hline & & & & 1.1 & & 0.2 & $\begin{array}{l}0.2 \\
1\end{array}$ & & $\begin{array}{l}0.2 \\
1\end{array}$ & 0.5 & & 0.2 & & 0.5 & & & 0.5 & 0.2 & 58.3 & & & 70 & $99-100$ \\
\hline 3 & 9 & & & 7 & 1 & & $\overline{7}$ & 9 & 1 & & & 1 & & & 1 & & & 1 & & & 1 & 485 & \\
\hline 0.6 & 1.9 & & & 1.4 & 0.2 & & 1.4 & 1.9 & 0.2 & & & 0.2 & & & 0.2 & & & 0.2 & & & 0.2 & & \\
\hline & & & & 1 & & & 7 & 3 & & & & & & & & 1 & & & & & & 217 & 99-101 \\
\hline & & & & 0.5 & & & 3.2 & 1.4 & & & & & & & & 0.5 & & & & & & & \\
\hline 1 & & & & & & 1 & 8 & 3 & & & & & & & & & & & & & & 341 & $99-101$ \\
\hline 0.2 & & & & & & 0.3 & 2.3 & 0.9 & & & & & & & & & & & & & & & \\
\hline 3 & & & & 5 & 1 & & 12 & 14 & 2 & & & & & & & & & & & & & 425 & $99-101$ \\
\hline 0.7 & & & & 1.2 & 0.2 & & 2.8 & 3.3 & 0.5 & & & & & & & & & & & & & & \\
\hline & & & & 4 & & & 8 & 2 & 1 & & & & & & & & & & & & & 317 & $89-91$ \\
\hline & & & & 1.3 & & & 2.5 & 0.6 & 0.3 & & & & & & & & & & & & & & \\
\hline 2 & 2 & & & 3 & & & 8 & 5 & & & & & & & & & & & & & & 439 & \\
\hline 0.5 & 0.5 & & & 0.7 & & & 1.8 & 1.1 & & & & & & & & & & & & & & & \\
\hline & & & & 16 & & & 2 & 6 & & & & & & 1 & 1 & & 3 & & 98 & & & 369 & $119-121$ \\
\hline & & & & 4.3 & & & 0.5 & 1.6 & & & & & & 0.2 & 0.2 & & 0.8 & & 26.6 & & & & \\
\hline & & & & 1 & & 1 & & & & & & & & & & & & & 243 & & & 446 & $99-104$ \\
\hline & & & & 0.2 & & 0.2 & 3.2 & & & & & & & & & & & & & & & 545 & \\
\hline & & & & & & & 1 & & & & & & & & & & & & 430 & & & 470 & \\
\hline & & & & & & & 0.2 & & & & & & & & & & & & 916 & & & & \\
\hline 1 & & & & 1 & & & & 3 & & & & & & & & & & & 422 & & & 522 & \\
\hline 0.2 & & & & 0.4 & & 0.2 & & 0.6 & & & & & & & & & & & 80.7 & & & & \\
\hline 1 & & & & & & & 1 & & & & 1 & & & & & & & & 188 & & & 427 & \\
\hline 0.2 & & & & & & & 0.2 & & & & 0.2 & & & & & & & & 44.4 & & & & \\
\hline 1 & & & & & & & 1 & & & & & & & & & & & & 399 & & & 459 & \\
\hline 0.2 & & & & & & & 0.2 & & & & & & & & & & & & 86.9 & & & & \\
\hline 1 & & & & & & & 1 & & & & & & & & & & & & 415 & & & 477 & \\
\hline 0.2 & & & & & & & 0.2 & & & & & & & & & & & & 87.0 & & & & \\
\hline & & & & & & & & & & & & & & & & & & & $\begin{array}{c}385 \\
81.5\end{array}$ & & & 472 & \\
\hline 3 & & & & & & & 1 & & & & & & & & & & & & 426 & & & 523 & \\
\hline 0.6 & & & & & & & 0.2 & & & & & & & & & & & & 81.4 & & & & \\
\hline & & & & & & & 1 & & & & & & & & & & & & 476 & & & 505 & \\
\hline & & & & & & & 0.2 & & & & & & & & & & & & 94.0 & & & & \\
\hline & & & & & & & 3 & 2 & & & & & & & & & & & 409 & & & 482 & \\
\hline & & & & & & & 0.6 & 0.4 & & & & & & & & & & & 85.0 & & & & \\
\hline & & & & & & & & & & & & & & & & & & & $\begin{array}{l}405 \\
93.2\end{array}$ & & & 435 & \\
\hline & & & & & & & & & & & & & & & & & & & 356 & & & 388 & \\
\hline 1 & & & & & & & & & & & & & & & & & & & $\begin{array}{c}92.0 \\
360\end{array}$ & & & 439 & \\
\hline 0.2 & & & & & & & & & & & & & & & & & & & 82.0 & & & & \\
\hline & & & & & & & 2 & & & & & & & & & & & & 234 & & & 462 & \\
\hline & & & & & & & 0.4 & & & & & & & & & & & & 51.0 & & & & \\
\hline & & & & & & & & & & & & & & & & & & & 328 & & & 405 & \\
\hline & & & & & & & & & & & & & & & & & & & 81.0 & & & & \\
\hline & & & & & & & & & & & 2 & & & & & & & & 475 & & & 540 & \\
\hline & & & & & & & & & & & 0.4 & & & & & & & & 88.0 & & & & \\
\hline 1 & & & & & & & 2 & & & & & & & & & & & & 469 & & & 522 & \\
\hline 0.2 & & & & & & & 0.4 & & & & & & & & & & & & 90.0 & & & & \\
\hline & & & & & & & 1 & & & & & & & & & & & & 48.1 & 1 & & 536 & \\
\hline & & & & & & & 0.2 & & & & & & & & & & & & 89.8 & 0.2 & & & \\
\hline 1 & & & & & & & 1 & & & & & & & & & & & & 315 & & & 469 & \\
\hline 0.2 & & & & & & & 0.2 & & & & & & & & & & & & 67.1 & & & & \\
\hline & & & & & & & & 1 & & & & & & & & & & & 401 & & & 473 & \\
\hline & & & & & & & & 0.2 & & & & & & & & & & & 84.9 & & & & \\
\hline & & & & & & & & 1 & & & & & & & & & & & 382 & & & 423 & \\
\hline & & & & & & & & 0.2 & & & & & & & & & & & 90.5 & & & & \\
\hline & & & & & & & & & & & & & & & & & & & $\begin{array}{c}413 \\
79.4\end{array}$ & & & 521 & \\
\hline & & & & & & & & & & & & & & & & & & & 237 & & & 461 & \\
\hline 1 & & & & & & & & & & & & & & & & & & & 51.3 & & & & \\
\hline $\begin{array}{l}1 \\
0.2\end{array}$ & & & & & & & & & & & & & & & & & & & 382 & & & 469 & \\
\hline $\begin{array}{l}0.2 \\
1\end{array}$ & & & & & & & & & & & & & & & & & & & 81.5 & & & & \\
\hline
\end{tabular}


TABLE 2A - Continued

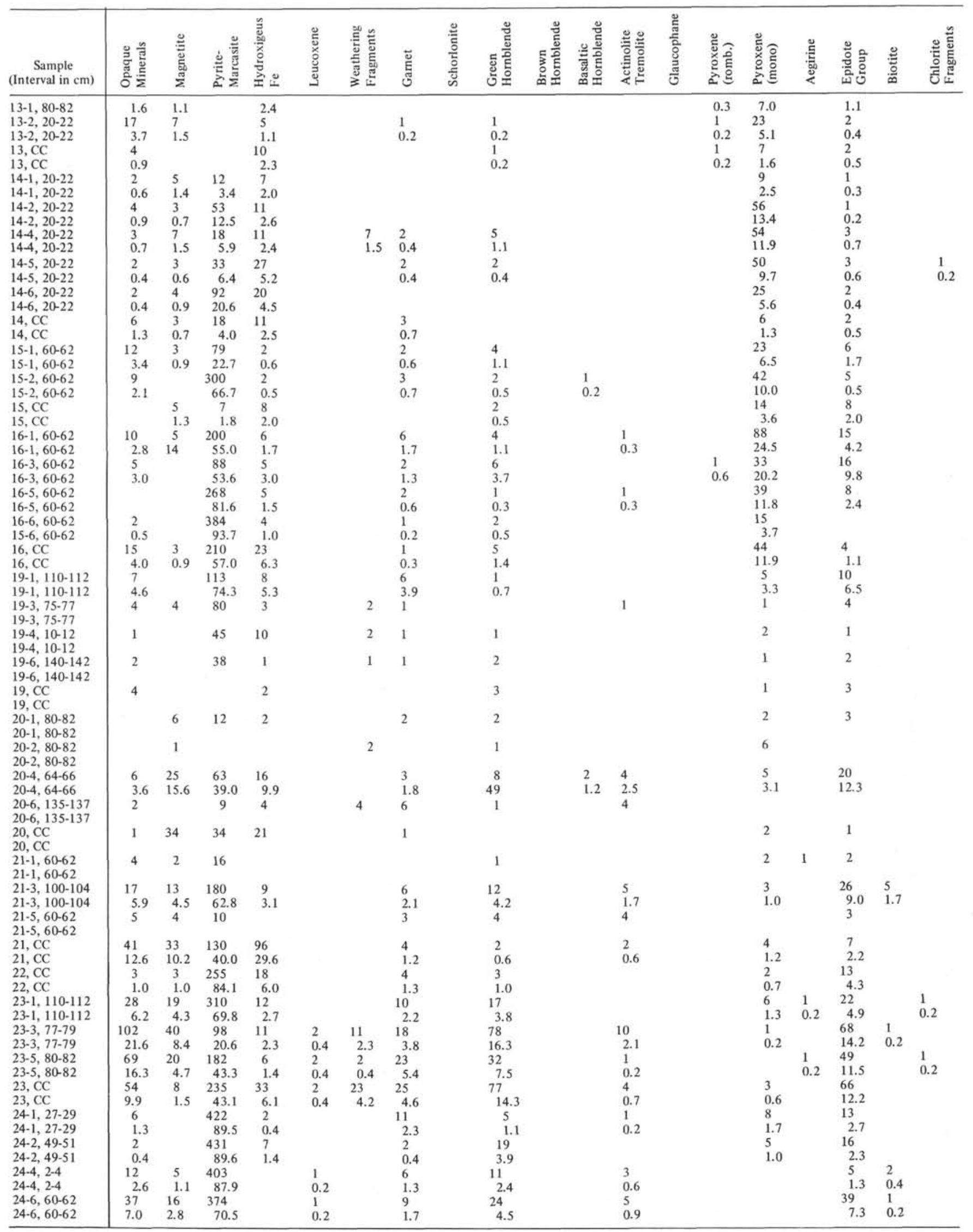


TABLE 2A - Continued

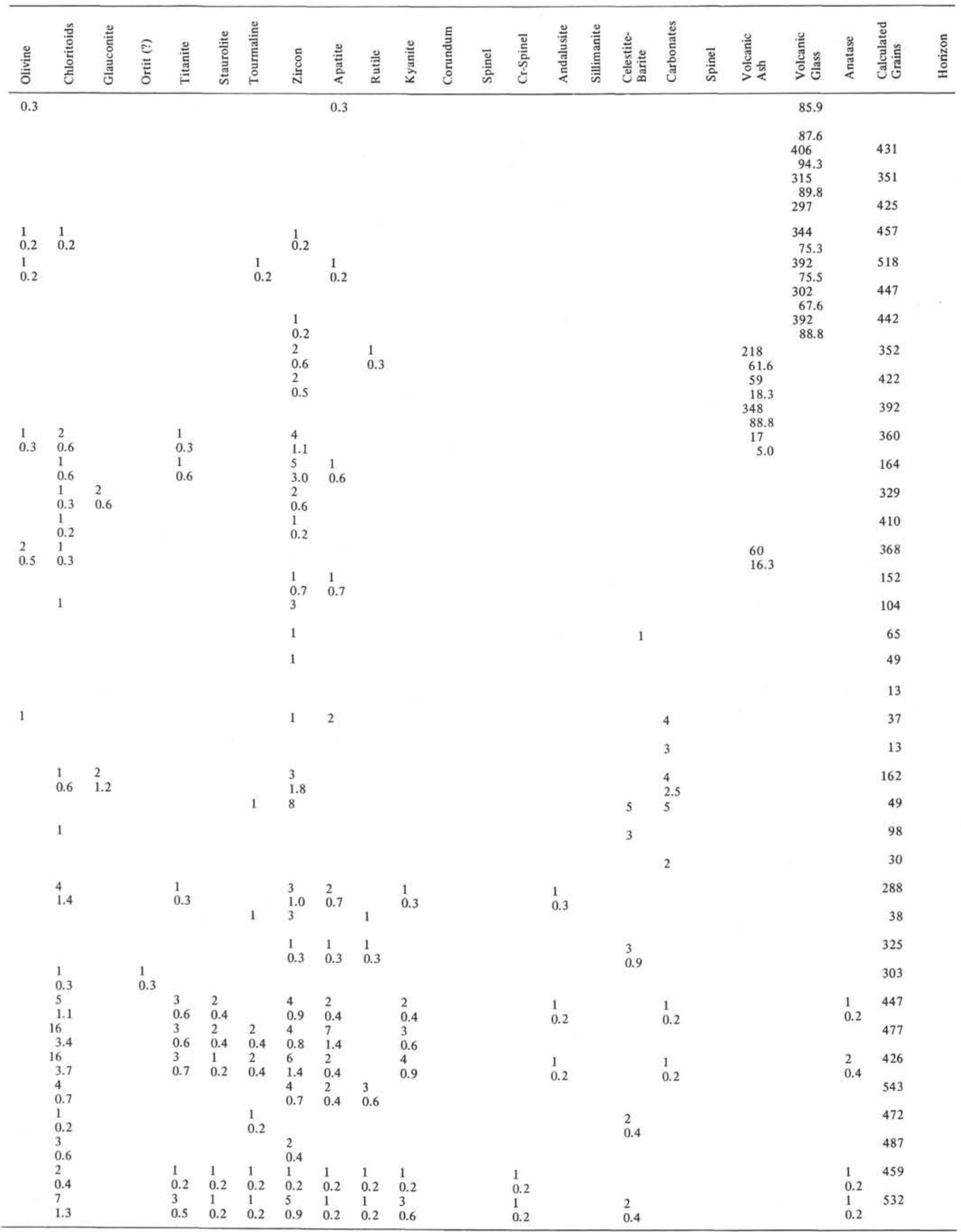


TABLE 2A - Continued

\begin{tabular}{|c|c|c|c|c|c|c|c|c|c|c|c|c|c|c|c|c|c|c|c|}
\hline $\begin{array}{c}\text { Sample } \\
\text { (Interval in } \mathrm{cm} \text { ) }\end{array}$ & 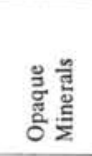 & 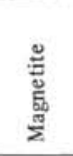 & 密 & 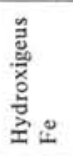 & 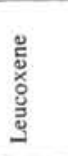 & 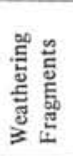 & 䓌 & 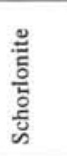 & 总 & ह⿸\zh14𠄌⿺乚一兀 & 苞 & 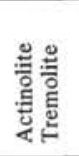 & 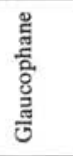 & 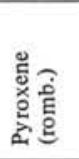 & 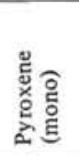 & 壳 & 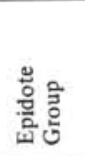 & 气̆ & 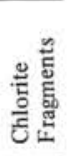 \\
\hline $24, \mathrm{CC}$ & 14 & & 344 & 21 & & & 9 & & 35 & & & 3 & & 1 & 4 & & 32 & & \\
\hline $24, \mathrm{CC}$ & 2.9 & & 71.5 & 4.3 & & & 1.9 & & 7.2 & & & 0.6 & & 0.2 & 0.8 & & 6.6 & & \\
\hline $25-1,64-66$ & 19 & 10 & 397 & 7 & 2 & & 9 & & 18 & & & 4 & & & & & 21 & 1 & 1 \\
\hline $25-1,64-66$ & 3.7 & 2.0 & 77.8 & 1.3 & 0.4 & & 1.7 & & 3.5 & & & 0.8 & & & & & 4.1 & 0.2 & 0.2 \\
\hline $25-3,44-46$ & 23 & 15 & 371 & 2 & 1 & 4 & 10 & & 2.6 & & & 1 & & 1 & 1 & 1 & 30 & & 2 \\
\hline $25-3,44-46$ & 4.5 & 2.9 & 73.0 & 0.4 & 0.2 & 0.8 & 1.9 & & 5.1 & & & 0.2 & & 0.2 & 0.2 & 0.2 & 5.8 & & 0.4 \\
\hline $25-4,75-77$ & 16 & 8 & 314 & 6 & 1 & & 11 & & 15 & & & 2 & & & 2 & 1 & 19 & 1 & 2 \\
\hline $25-4,75-77$ & 3.8 & 2.0 & 76.4 & 1.4 & 0.2 & & 2.6 & & 3.6 & & & 0.5 & & & 0.5 & 0.2 & 4.6 & 0.2 & 0.5 \\
\hline $25-6,71-73$ & 31 & 12 & 399 & 6 & 1 & & 6 & & 21 & & & 3 & & & & & 23 & & \\
\hline $25-6,71-73$ & 6.0 & 2.3 & 76.5 & 1.1 & 0.2 & & 1.1 & & 4.0 & & & 0.6 & & & & & 4.4 & & \\
\hline $25, \mathrm{CC}$ & 35 & 6 & 363 & 10 & & 14 & 32 & 1 & 58 & & & 4 & & & 5 & & 40 & & \\
\hline $25, \mathrm{CC}$ & 5.9 & 1.0 & 61.1 & 1.7 & & 2.3 & 5.4 & 0.2 & 9.8 & & & 0.7 & & & 0.8 & & 6.7 & & \\
\hline $26-1,58-60$ & 35 & 2 & 316 & 2 & & 7 & 6 & & 11 & & & 2 & & & 1 & & 26 & & \\
\hline $26-1,58-60$ & 84 & 0.5 & 75.5 & 0.5 & & 1.7 & 1.4 & & 2.6 & & & 0.5 & & & 0.2 & & 6.1 & & \\
\hline $26-3,68-70$ & 61 & 7 & 320 & 3 & & 10 & 8 & & 16 & & & 1 & & & 1 & & 31 & & \\
\hline $26-3,68-70$ & 13.0 & 1.5 & 68.5 & 0.6 & & 2.1 & 1.7 & & 3.4 & & & 0.2 & & & 0.2 & & 6.7 & & \\
\hline $26-4,39-41$ & 40 & 8 & 297 & 3 & 2 & 8 & 24 & & 26 & & & 3 & & & 2 & & 30 & & \\
\hline $26-4,39-41$ & 8.9 & 1.8 & 66.2 & 0.7 & 0.4 & 1.8 & 5.3 & & 5.7 & & & 0.7 & & & 0.4 & & 6.7 & & \\
\hline $26, \mathrm{CC}$ & 50 & 6 & 286 & 4 & & 17 & 25 & & 55 & & & 4 & & & 6 & & 47 & & 5 \\
\hline $26, \mathrm{CC}$ & 9.6 & 1.1 & 54.2 & 0.8 & & 3.2 & 4.8 & & 10.5 & & & 0.8 & & & 1.1 & & 8.9 & & 1.0 \\
\hline $27-1,92-96$ & 93 & 4 & 217 & 2 & 4 & 25 & 15 & & 54 & & & 2 & & & 3 & & 72 & & \\
\hline $27-1,92-96$ & 18.1 & 0.8 & 42.8 & 0.4 & 0.8 & 4.9 & 2.9 & & 10.6 & & & 0.4 & & & 0.6 & & 14.3 & & \\
\hline $27-2,103-105$ & 52 & 5 & 244 & 3 & & 18 & 6 & & 36 & & & 3 & & & 5 & & 51 & & \\
\hline $27-2,103-105$ & 11.2 & 1.1 & 52.9 & 0.7 & & 3.9 & 1.3 & & 7.8 & & & 0.7 & & & 1.1 & & 11.0 & & \\
\hline $27-3,21-23$ & 78 & 5 & 257 & 5 & & 16 & 26 & & 24 & & & 4 & & & 3 & & 39 & 1 & \\
\hline $27-3,21-23$ & 16.1 & 1.0 & 53.1 & 1.0 & & 3.3 & 5.3 & & 5.0 & & & 0.8 & & & 0.6 & & 8.1 & 0.2 & \\
\hline $28-1,75-77$ & 54 & 5 & 311 & 5 & 3 & 10 & 14 & & 28 & & & 1 & & & 5 & & 48 & & \\
\hline $28-1,75-77$ & 10.7 & 1.0 & 61.9 & 1.0 & 0.6 & 2.0 & 2.8 & & 5.5 & & & 0.2 & & & 1.0 & & 9.5 & & \\
\hline $28-2,129-131$ & 85 & 1 & 219 & 4 & & 17 & 28 & & 16 & & & & & & 4 & & 42 & 1 & \\
\hline $28-2,129-131$ & 19.7 & 0.2 & 50.4 & 0.9 & & 3.9 & 6.5 & & 3.7 & & & & & & 0.9 & & 9.7 & 0.2 & \\
\hline $28, \mathrm{CC}$ & 56 & 15 & 181 & 10 & & 20 & 32 & & 49 & & & 4 & & & 9 & & 66 & & \\
\hline $28, \mathrm{CC}$ & 11.8 & 3.2 & 38.6 & 2.1 & & 4.2 & 6.8 & & 10.5 & & & 0.8 & & & 1.9 & & 14.0 & & \\
\hline $29-2,124-126$ & 39 & 8 & 340 & 5 & & 14 & 15 & & 12 & & & 5 & 1 & & & & 40 & & \\
\hline $29-2,124-126$ & 7.6 & 1.6 & 66.6 & 1.0 & & 2.7 & 2.9 & & 2.3 & & & 1.0 & 0.2 & & & & 7.8 & & \\
\hline $29-3,27-29$ & 44 & 2 & 378 & 4 & & 14 & 14 & & 5 & & & & & & 1 & & 36 & & \\
\hline $29-3,27-29$ & 8.5 & 0.4 & 72.8 & 0.8 & & 2.7 & 2.7 & & 1.0 & & & & & & 0.2 & & 7.0 & & \\
\hline $29-4,24-26$ & 60 & 6 & 274 & 5 & & 5 & 21 & & 36 & & & 2 & & & 4 & & 63 & 1 & \\
\hline $29-4,24-26$ & 12.1 & 1.2 & 55.0 & 1.0 & & 1.0 & 4.2 & & 7.2 & & & 0.4 & & & 0.8 & & 12.7 & 0.2 & \\
\hline $29-5,89-91$ & 81 & 5 & 189 & 10 & & 9 & 13 & & 32 & & & 3 & & & 7 & & 83 & & \\
\hline $29-5,89-91$ & 17.6 & 1.1 & 42.0 & 2.2 & & 2.0 & 28 & & 7.0 & & & 0.7 & & & 1.5 & & 18.3 & & \\
\hline $29, \mathrm{CC}$ & 65 & 11 & 237 & 16 & 2 & 23 & 23 & & 42 & & & 5 & & & 7 & & 59 & & \\
\hline $29, \mathrm{CC}$ & 12.5 & 2.1 & 45.5 & 3.1 & 0.4 & 4.4 & 4.4 & & 8.1 & & & 1.0 & & & 1.4 & & 11.4 & & \\
\hline $30-1,76-78$ & 40 & 2 & 432 & 5 & & & 5 & & 4 & & & & & & 3 & & 31 & & \\
\hline $30-1,76-68$ & 7.4 & 0.4 & 81.0 & 0.9 & & & 0.9 & & 0.7 & & & & & & 0.6 & & 5.8 & & \\
\hline $30-2,25-27$ & 122 & 4 & 106 & 8 & 2 & 31 & 28 & 2 & 41 & & & 1 & & & 5 & 1 & 50 & & 1 \\
\hline $30-2,25-27$ & 27.7 & 0.9 & 23.5 & 1.8 & 0.4 & 6.9 & 6.2 & 0.4 & 9.1 & & & 0.2 & & & 1.1 & 0.2 & 11.2 & & 0.2 \\
\hline $30-3,62-64$ & 107 & 8 & 305 & 4 & & 12 & 18 & & 31 & & & 5 & & & 5 & & 37 & 1 & \\
\hline $30-3,62-64$ & 19.3 & 1.4 & 54.9 & 0.7 & & 2.1 & 3.2 & & 5.6 & & & 1.0 & & & 1.0 & & 6.7 & 0.2 & \\
\hline $30-5,52-54$ & 70 & 2 & 165 & 12 & 2 & 20 & 29 & & 84 & & & 2 & & & 14 & & 75 & 1 & \\
\hline $30-5,52-54$ & 14.1 & 0.4 & 33.2 & 2.4 & 0.4 & 4.0 & 5.8 & & 17.0 & & & 0.4 & & & 2.8 & & 15.1 & 0.2 & \\
\hline $30-6,18-20$ & 117 & 5 & 165 & 10 & 3 & 17 & 25 & & 55 & & & 4 & & 1 & 8 & & 65 & & \\
\hline $30-6,18-20$ & 23.7 & 1.0 & 33.5 & 2.0 & 0.6 & 3.4 & 5.1 & & 11.2 & & & 0.8 & & 0.2 & 1.6 & & 13.2 & & \\
\hline $31-1,71-73$ & 60 & 7 & 251 & 11 & & 16 & 27 & & 45 & & & 3 & & & 3 & & 53 & 1 & \\
\hline $31-1,71-73$ & 11.9 & 1.4 & 49.8 & 2.3 & & 5.2 & 5.3 & & 9.0 & & & 0.6 & & & 0.6 & & 10.5 & 0.2 & \\
\hline $31-2,72-74$ & 241 & 15.0 & 54 & 18 & 2 & 20 & 28 & & 45 & & & 4 & & & 19 & & 72 & & \\
\hline $31-2,72-74$ & 45.0 & 2.8 & 10.1 & 3.3 & 0.3 & 3.7 & 5.2 & & 8.4 & & & 0.8 & & & 3.5 & & 13.5 & & \\
\hline $31-3,62-64$ & 269 & 44 & 72 & 16 & & 18 & 26 & 1 & 15 & & & 2 & & & 10 & & 30 & & \\
\hline $31-3,62-64$ & 51.6 & 8.4 & 13.8 & 3.0 & & 3.6 & 5.0 & 0.2 & 2.8 & & & 0.3 & & & 1.9 & & 5.7 & & \\
\hline $31-4,48-50$ & 53 & 4 & 169 & 10 & & 23 & 11 & 1 & 42 & & & 5 & & & 7 & & 64 & 5 & \\
\hline $31-4,48-50$ & 12.8 & 1.0 & 40.6 & 2.3 & & 5.5 & 2.6 & 0.2 & 10.2 & & & 1.2 & & & 1.7 & & 15.4 & 1.2 & \\
\hline $32-2,82-85$ & 107 & 15 & 72 & 24 & 2 & 31 & 15 & 1 & 78 & 1 & & 4 & & 2 & 19 & & 49 & 1 & 1 \\
\hline $32-2,82-85$ & 24.2 & 3.4 & 16.3 & 5.4 & 0.5 & 7.0 & 3.4 & 0.2 & 17.6 & 0.2 & & 0.9 & & 0.5 & 4.3 & & 11.0 & 0.2 & 0.2 \\
\hline
\end{tabular}

almost complete alteration of the glass into montmorillonite, and the absence of an admixture of acid volcanogenic material. This results in a partial enrichment of the large size fractions by terrigenous components.

\section{Unit 2}

The sediments of Unit 2 (middle Miocene-Pliocene) are distinct in composition and in the content of various genetic components. Biogenic components in these sediments show a remarkable increase in content. Individual thin interbeds are characterized by a sharp predominance of diatom skeletons. However, only in these interbeds are they considered as a major sediment component (Figures 3 and 4). There is also a large increase in the quantity of carbonate biogenic material, mainly foraminifera tests.

The silt fractions in Unit 2 have a higher content of volcanogenic material as compared to Unit 1 . The main portion of this material is represented by devitrified volcanic glass. However, there is also a considerable 


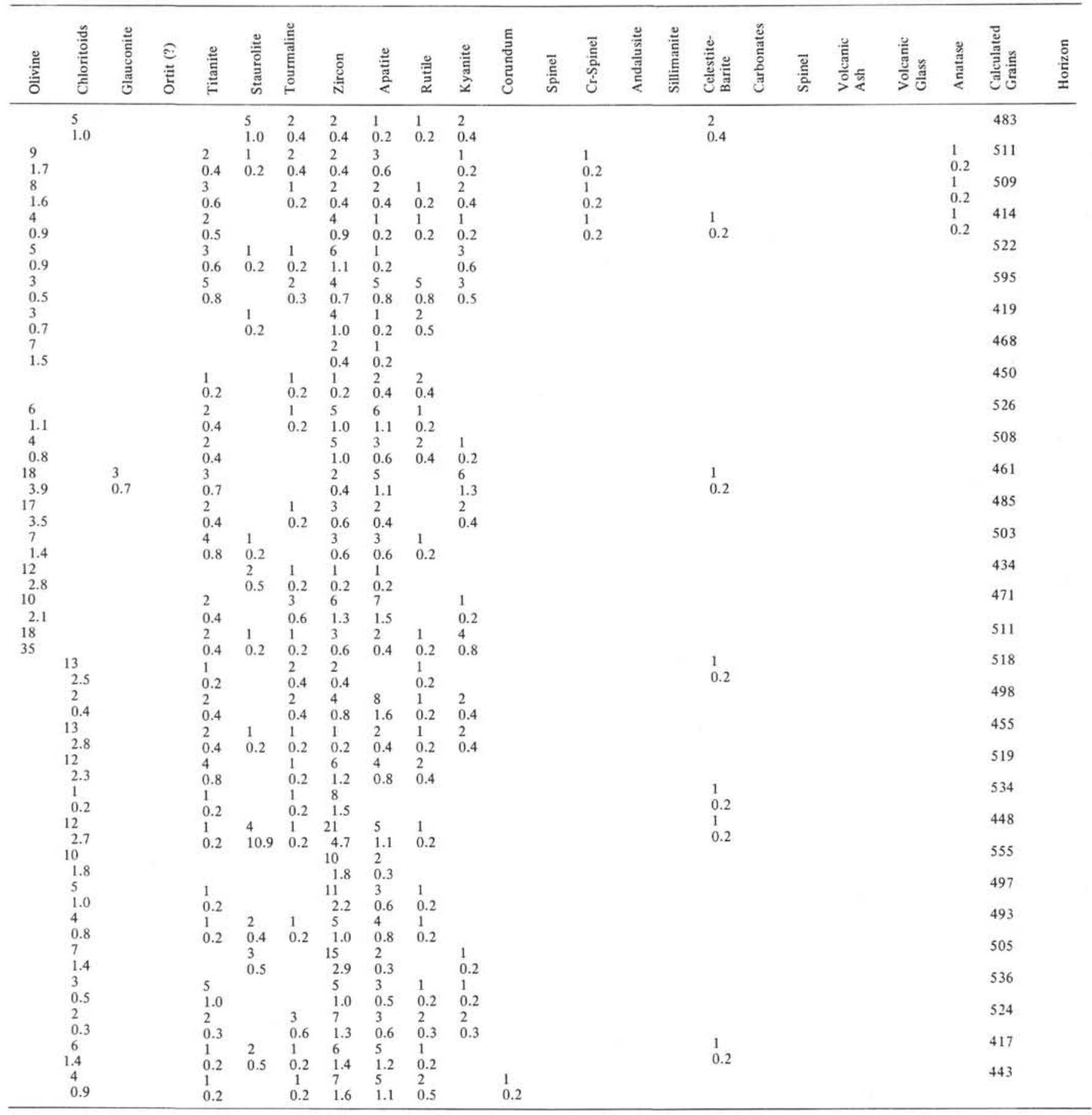

amount of unaltered glass present. It is predominantly basic, but also includes acid glasses. The amount of acid glass increases regularly upward in Unit 2 . The maximum amount of the acid glass (up to a third of the total bulk of glass) is recorded in Pliocene sediments.

Distribution of authigenic components in the silt fraction of Unit 2 is very irregular. At its lower portion (at the boundary with early Miocene), the unit is characterized by a high content of iron sulfides and zeolites. The quantity of these components decreases sharply towards the upper boundary of Unit 2.
A common mineral in the clay fraction (less than 1 $\mu \mathrm{m})$ is montmorillonite. The content tends to generally decrease in the upper layers of the unit. This can be explained by the fact that in the younger sediments, a part of the volcanogenic ash material has not yet decomposed, although it is nearly devitrified. Also, the acid glasses are not strongly altered. There is also a considerable decrease of finely dispersed zeolites in the sediments of Unit 2.

Apart from the clay composition, Unit 2 differs from Unit 3 by a gradual increase of hydromica and probable 


\begin{tabular}{|c|c|c|c|c|c|c|c|c|c|c|c|c|c|c|c|c|c|c|c|c|}
\hline $1-1,70-72$ & 61 & 26 & 10 & 2 & & & & & 1 & 89 & 12 & 12 & & 2 & & & & 41 & & 256 \\
\hline $1-1,70-72$ & 23.8 & 10.2 & 3.9 & 0.8 & & & & & 0.4 & 34.8 & 4.7 & 4.7 & & 0.8 & & & & 15.9 & & \\
\hline $1-3,100-104$ & 75 & 56 & 20 & & 1 & & & & 5 & 10 & & 1 & & & & & & 51 & & 219 \\
\hline $1-3,100-104$ & 34.3 & 25.5 & 9.1 & & 0.5 & & & & 2.3 & 4.5 & & 0.5 & & & & & & 23.3 & & \\
\hline $1-4,100-102$ & 80 & 31 & 15 & 1 & 2 & 1 & & & & 227 & 69 & 10 & 1 & 2 & & 4 & & & & 443 \\
\hline $1-4,100-102$ & 18.1 & 7.0 & 3.4 & 0.2 & 0.4 & 0.2 & & & & 51.3 & 15.6 & 2.3 & 0.2 & 0.4 & & 0.9 & & & & \\
\hline $1-5,90-92$ & 220 & 85 & 11 & 4 & 4 & 1 & 2 & & 1 & 19 & 12 & 1 & & 1 & & 6 & & 36 & & 404 \\
\hline $1-5,90-92$ & 54.5 & 21.5 & 2.7 & 0.9 & 0.9 & 0.2 & 0.4 & & 0.2 & 4.7 & 3.0 & 0.2 & & 0.2 & & 15 & 0.2 & 8.9 & & \\
\hline $1, \mathrm{CC}$ & 76 & 27 & 19 & 3 & & & & & 1 & 53 & & 1 & & & & & & 42 & 38 & 260 \\
\hline $1, \mathrm{CC}$ & 29.2 & 10.4 & 7.3 & 1.2 & & & & & 0.4 & 20.4 & & 0.4 & & & & & & 16.1 & 14.6 & \\
\hline $2-1,98-100$ & 175 & 48 & 13 & 3 & 1 & & & 1 & 1 & 69 & 16 & 7 & & 2 & 1 & 12 & & 53 & & 402 \\
\hline $2-1,98-100$ & 43.5 & 12.0 & 3.2 & 0.7 & 0.2 & & & 0.2 & 0.2 & 17.4 & 4.0 & 1.7 & & 0.5 & 0.2 & 3.0 & & 13.2 & & \\
\hline $2-2,50-52$ & 134 & 49 & 15 & 1 & 1 & & 2 & 1 & 1 & 19 & 20 & 20 & 1 & 2 & 2 & 7 & 1 & 88 & 1 & 366 \\
\hline $2-2,50-52$ & 36.5 & 13.4 & 4.1 & 0.3 & 0.3 & & 0.5 & 0.3 & 0.3 & 5.2 & 5.5 & 5.5 & 0.3 & 0.5 & 0.5 & 1.9 & 0.3 & 24.0 & 0.3 & \\
\hline $2-4,99-101$ & 71 & 52 & 12 & 1 & 1 & & 2 & & 4 & 7 & & & & 3 & 5 & & & 53 & & 211 \\
\hline $2-4,99-101$ & 33.6 & 24.6 & 5.7 & 0.5 & 0.5 & & 1.0 & & 1.9 & 3.3 & & & & 1.4 & 2.4 & & & 25.1 & & \\
\hline $2, \mathrm{CC}$ & 58 & 25 & 9 & 1 & 1 & & 4 & & & 24 & & & & & & & & 18 & 71 & 211 \\
\hline $2, \mathrm{CC}$ & 27.4 & 11.8 & 4.3 & 0.5 & 0.5 & & 1.9 & & & 11.3 & & & & & & & & 8.5 & 33.8 & \\
\hline $3-1,99-101$ & 73 & 57 & 21 & 2 & 1 & & 7 & & & 3 & & & & 1 & 1 & & & 78 & & 244 \\
\hline $3-1,99-101$ & 30.0 & 23.3 & 8.6 & 0.8 & 0.4 & & 2.9 & & & 1.2 & & & & 0.4 & 0.4 & & & 32.0 & & \\
\hline $3-3,99-101$ & 66 & 42 & 19 & 6 & & & 4 & & & 5 & & & & & & & & 76 & & 218 \\
\hline $3-3,99-101$ & 30.2 & 19.3 & 8.7 & 2.7 & & & 1.8 & & & 2.3 & & & & & & & & 35.0 & & \\
\hline $3-4,99-101$ & 78 & 42 & 18 & 4 & 3 & & 3 & & & & & & & & & & & 53 & & 201 \\
\hline $3-4,99-101$ & 38.8 & 20.8 & 9.0 & 2.0 & 1.5 & & 1.5 & & & & & & & & & & & 26.4 & & \\
\hline $3-5,89-91$ & 94 & 48 & 20 & 5 & 1 & & 1 & & & 4 & & & & & & & & 51 & & 224 \\
\hline $3-5,89-91$ & 42.2 & 21.4 & 8.8 & 2.2 & 0.4 & & 0.4 & & & 1.8 & & & & & & & & 22.8 & & \\
\hline $3, \mathrm{CC}$ & 66 & 48 & 15 & 2 & & & 1 & & 2 & & & 2 & & & & & & 75 & 44 & 255 \\
\hline $3, \mathrm{CC}$ & 25.9 & 18.7 & 5.8 & 0.8 & & & 0.4 & & 0.8 & & & 0.8 & & & & & & 29.5 & 17.3 & \\
\hline $4-1,119-121$ & 167 & 55 & 35 & 2 & 3 & & 3 & & & 1 & 10 & 15 & & 4 & & & & 75 & & 370 \\
\hline $4-1,119-121$ & 45.2 & 14.8 & 9.5 & 0.5 & 0.8 & & 0.8 & & & 0.2 & 2.7 & 4.1 & & 1.1 & & & & 20.3 & & \\
\hline $4-2,99-104$ & 133 & 47 & 37 & 19 & 3 & 1 & 1 & 1 & 1 & 1 & 5 & 2 & & 4 & & 1 & & 106 & & 362 \\
\hline $4-2,99-104$ & 36.8 & 13.0 & 10.2 & 5.2 & 0.8 & 0.3 & 0.3 & 0.3 & 0.3 & 0.3 & 1.5 & 0.5 & & 1.1 & & 0.3 & & 29.1 & & \\
\hline $5-1,99-100$ & 19 & 19 & 2 & 8 & & & 1 & & & & 107 & 9 & & 88 & & & & & & 253 \\
\hline $5-1,99-100$ & 7.5 & 7.5 & 0.8 & 3.2 & & & 0.4 & & & & 42.3 & 3.5 & & 34.8 & & & & & & \\
\hline $5-2,139-141$ & 57 & 30 & & 4 & & & 1 & & & 1 & 85 & 11 & & 27 & & & & & & 216 \\
\hline $5-2,139-141$ & 26.5 & 13.8 & & 1.8 & & & 0.5 & & & 0.5 & 39.4 & 51 & & 12.4 & & & & & & \\
\hline $5, \mathrm{CC}$ & 36 & 32 & 7 & 2 & 1 & & & & 2 & & 25 & 24 & 1 & 29 & & & & 46 & & 205 \\
\hline $5, \mathrm{CC}$ & 17.5 & 15.6 & 3.4 & 1.0 & 0.5 & & & & 1.0 & & 12.3 & 11.7 & 0.5 & 14.1 & & & & 22.4 & & \\
\hline $6-1,139-141$ & 51 & 26 & 9 & 5 & & & & & 1 & 7 & 78 & 2 & & 73 & & & & & & 252 \\
\hline $6-1,139-141$ & 20.3 & 10.6 & 3.6 & 2.0 & & & & & 0.4 & 2.8 & 30.4 & 0.8 & & 29.1 & & & & & & \\
\hline $6-2,29-31$ & 9 & 3 & 2 & 4 & & & & 2 & & & 115 & 21 & & 77 & & & & & & 233 \\
\hline $6-2,29-31$ & 3.9 & 1.3 & 0.9 & 1.7 & & & & 0.9 & & & 48.8 & 9.1 & & 33.4 & & & & & & \\
\hline $6-4,84-86$ & 12 & 12 & 1 & 11 & & & 1 & & & 3 & 110 & 39 & & 86 & & & & & & 275 \\
\hline $6-4,84-86$ & 4.3 & 4.3 & 0.4 & 4.0 & & & 0.4 & & & 1.1 & 40.3 & 14.2 & & 31.0 & & & & & & \\
\hline $6-5,73-75$ & 14 & 4 & 2 & 3 & & & & & & & 105 & 19 & & 74 & & & & & & 221 \\
\hline $6-5,73-75$ & 6.3 & 1.8 & 0.9 & 1.4 & & & & & & & 47.8 & 8.6 & & 33.2 & & & & & & \\
\hline $6, \mathrm{CC}$ & 5 & 4 & & 3 & & & & & & 1 & 98 & & & 116 & & & & & 2 & 229 \\
\hline $6, \mathrm{CC}$ & 2.2 & 1.7 & & 1.3 & & & & & & 0.4 & 43.0 & & & 50.5 & & & & & 0.9 & \\
\hline
\end{tabular}




\begin{tabular}{|c|c|c|c|c|c|c|c|c|c|c|c|c|c|c|c|c|c|c|c|c|c|}
\hline $7-1,74-76$ & 75 & 22 & 13 & 8 & & 2 & & 4 & & & & 145 & 46 & 4 & 100 & & & 2 & 39 & & 460 \\
\hline $7-1,74-76$ & 16.3 & 4.8 & 2.8 & 1.7 & & 0.4 & & 0.9 & & & & 31.3 & 10.0 & 0.9 & 22.0 & & & 0.4 & 8.5 & & \\
\hline $7-3,74-76$ & 6 & 4 & 5 & 6 & & 1 & & 2 & & & & 152 & 28 & 4 & 140 & & & 1 & 5 & & 354 \\
\hline $7-3,74-76$ & 1.7 & 1.4 & 1.4 & 1.7 & & 0.2 & & 0.5 & & & & 43.0 & 7.9 & 1.1 & 39.5 & & & 0.2 & 1.4 & & \\
\hline $7-4,75-77$ & 5 & 3 & 5 & 12 & 1 & 3 & & 23 & & & & 160 & 86 & 3 & 43 & & & & 7 & & 351 \\
\hline $7-4,75-77$ & 1.4 & 0.8 & 1.4 & 3.4 & 0.2 & 0.8 & & 6.6 & & & & 45.7 & 24.7 & 0.8 & 12.2 & & & & 2.0 & & \\
\hline $7, \mathrm{CC}$ & 9 & 4 & 3 & 3 & & & & & 2 & & & 59 & 80 & & 48 & & & & & 13 & 221 \\
\hline $7, \mathrm{CC}$ & 4.1 & 1.8 & 1.4 & 1.4 & & & & & 0.9 & & & 26.8 & 36.0 & & 21.8 & & & & & 5.8 & \\
\hline $8-2,75-77$ & 6 & 2 & 2 & 13 & & & & & & & & 108 & 176 & 1 & 58 & & & 1 & & & 367 \\
\hline $8-2,75-77$ & 1.6 & 0.5 & 0.5 & 3.6 & & & & & & & & 29.5 & 48.1 & 0.2 & 15.8 & & & 0.2 & & & \\
\hline $8-3,75-77$ & 13 & 4 & 2 & 15 & & 4 & & 1 & & & & 177 & 106 & 1 & 64 & 1 & & 6 & & & 394 \\
\hline $8-3,75-77$ & 3.3 & 1.0 & 0.5 & 3.8 & & 1.0 & & 0.2 & & & & 45.0 & 27.0 & 0.2 & 16.3 & 0.2 & & 1.5 & & & \\
\hline $9-1,139-140$ & 1 & 1 & 6 & 19 & & 1 & & 2 & & & & 238 & 99 & 2 & 113 & & & & & & 482 \\
\hline $9-1,139-140$ & 0.2 & 0.2 & 1.2 & 3.9 & & 0.2 & & 0.4 & & & & 49.4 & 20.6 & 0.4 & 23.5 & & & & & & \\
\hline $9-2,75-77$ & 2 & 1 & 5 & 13 & & 2 & & 1 & & & & 165 & 122 & & 54 & & & 1 & & & 366 \\
\hline $9-2,75-77$ & 0.5 & 0.2 & 1.4 & 3.5 & & 0.5 & & 0.2 & & & & 45.2 & 33.6 & & 14.7 & & & 0.2 & & & \\
\hline $9-5,75-77$ & 1 & & 2 & 22 & & 3 & & 3 & & & & 179 & 80 & 12 & 64 & & & 7 & & & 376 \\
\hline $9-5,75-77$ & 0.3 & & 0.5 & 5.8 & & 0.8 & & 0.8 & & & & 47.6 & 21.4 & 3.2 & 17.0 & & & 1.8 & & & \\
\hline $9, \mathrm{CC}$ & 1 & 2 & & 4 & & & & & 1 & 1 & & 45 & 96 & 1 & 50 & & & & 3 & 24 & 228 \\
\hline $9, \mathrm{CC}$ & 0.4 & 0.9 & & 1.7 & & & & & 0.4 & 0.4 & & 19.7 & 42.3 & 0.4 & 29.0 & & & & 1.3 & 10.5 & \\
\hline $11-1,80-82$ & 1 & & 2 & 3 & & 3 & & 5 & & 1 & & 153 & 32 & 1 & 155 & & & & & & 356 \\
\hline $11-1,80-82$ & 0.3 & & 0.6 & 0.8 & & 0.8 & & 1.4 & & 0.3 & & 43.0 & 9.0 & 0.3 & 43. & & & & & & \\
\hline $11-2,80-82$ & 1 & & 3 & 5 & & 1 & & 2 & & & & 152 & 44 & 2 & 137 & & & & & & 347 \\
\hline $11-2,80-82$ & 0.3 & & 0.9 & 1.4 & & 0.3 & & 0.6 & & & & 44.0 & 12.7 & 0.6 & 39.2 & & & & & & \\
\hline $11-4,70-72$ & 1 & & 1 & 6 & & 1 & & & & & & 228 & 12 & 1 & 306 & & & 1 & & & 557 \\
\hline $11-4,70-72$ & 0.2 & & 0.2 & 1.0 & & 0.2 & & & & & & 41.0 & 2.0 & 0.2 & 55.0 & & & 0.2 & & & \\
\hline $12-2,60-62$ & & & & 3 & & 1 & & & & & & 198 & 27 & & 249 & & & & & & 478 \\
\hline $12-2,60-62$ & & & & 0.6 & & 0.2 & & & & & & 41.4 & 5.6 & & 52.2 & & & & & & \\
\hline $12-4,20-22$ & 2 & & 1 & 2 & & & & & & & & 195 & 17 & 1 & 208 & & & & & & 426 \\
\hline $12-4,20-22$ & 0.5 & & 0.2 & 0.5 & & & & & & & & 46.0 & 4.0 & 0.2 & 48.6 & & & & & & \\
\hline $13-1,80-82$ & 2 & 2 & & 5 & & & & & & & & 90 & 31 & & 94 & & & & & & 224 \\
\hline $13-1,80-82$ & 0.9 & 0.9 & & 2.2 & & & & & & & & 40.2 & 13.8 & & 42.0 & & & & & & \\
\hline $13-2,20-22$ & & 1 & & 6 & & & & 2 & & & & 112 & 26 & & 90 & & & & & & 237 \\
\hline $13-2,20-22$ & & 0.4 & & 2.5 & & & & 0.8 & & & & 47.3 & 11.0 & & 38.0 & & & & & & \\
\hline $13, \mathrm{CC}$ & 2 & & & 12 & & & & & & & & 99 & 43 & 1 & 81 & & & & & 1 & 239 \\
\hline $13, \mathrm{CC}$ & 0.8 & & & 5.0 & & & & & & & & 41.5 & 17.9 & 0.4 & 34.0 & & & & & 0.4 & \\
\hline $14-1,20-22$ & 2 & & & 2 & & 3 & & & & & & 61 & 33 & & 110 & & 1 & & & & 212 \\
\hline $14-1,20-22$ & 0.9 & & & 0.9 & & 1.4 & & & & & & 28.7 & 15.6 & & 52.0 & & 0.5 & & & & \\
\hline $14-2,20-22$ & 1 & 2 & & 2 & & & 1 & & & 1 & & 90 & 18 & & 103 & & & & & & 218 \\
\hline $14-2,20-22$ & 0.5 & 0.9 & & 0.9 & & & 0.5 & & & 0.5 & & 413 & 8.2 & & 47.2 & & & & & & \\
\hline $14-4,20-22$ & 3 & 1 & 1 & 5 & & 1 & & & & 1 & & 79 & 32 & & 150 & & & & & & 273 \\
\hline $14-4,20-22$ & 10 & 0.4 & 0.4 & 1.8 & & 0.4 & & & & 0.4 & & 29.0 & 11.6 & & 55.0 & & & & & & \\
\hline $14-5,20-22$ & 3 & 1 & & 5 & & & & & & & & 124 & 41 & & 112 & & & & & & 286 \\
\hline $14-5,20-22$ & 1.0 & 0.4 & & 1.7 & & & & & & & & 43.6 & 14.4 & & 38.9 & & & & & & \\
\hline $14-6,20-22$ & 1 & & & 5 & & & & & & & & 115 & 14 & & 109 & & & & 4 & & 248 \\
\hline $14-6,20-22$ & 0.4 & & & 2.0 & & & & & & & & 46.0 & 5.5 & & 44.5 & & & & 1.6 & & \\
\hline $14, \mathrm{CC}$ & 3 & & & & 1 & & & & & & & 63 & 12 & & 127 & & & & & & 253 \\
\hline $14, \mathrm{CC}$ & 1.2 & & & & 0.4 & & & & & & & 25.0 & 4.8 & & 50.0 & & & & & & \\
\hline $15-1,60-62$ & 6 & 5 & & 5 & & & & 2 & & & & 105 & 11 & & 153 & & & & 5 & & 292 \\
\hline $15-1,60-62$ & 2.0 & 1.7 & & 1.7 & & & & 0.7 & & & & 36.0 & 38 & & 52.4 & & & & 1.7 & & \\
\hline $15-2,60-62$ & 8 & 2 & & 1 & 1 & 2 & & 3 & & 2 & 2 & 107 & 13 & & 97 & & & & & & 241 \\
\hline $15-2,60-62$ & 3.3 & 0.8 & & 1.7 & 0.4 & 0.8 & & 1.2 & & 0.8 & 0.8 & 44.6 & 5.4 & & 40.2 & & & & & & \\
\hline $15, \mathrm{CC}$ & 1 & 1 & & 3 & & & & & & & & 87 & 3 & & 167 & & & & & 13 & 275 \\
\hline $15, \mathrm{CC}$ & 0.4 & 0.4 & & 1.1 & & & & & & & & 31.3 & 1.1 & & 61.0 & & & & & 4.7 & \\
\hline $16-1,60-62$ & 9 & & & 4 & & 2 & 1 & 2 & & & & 80 & 44 & 3 & 93 & & & & & & 238 \\
\hline $16-1,60-62$ & 3.8 & & & 1.7 & & 0.8 & 0.4 & 0.8 & & & & 33.7 & 18.5 & 1.3 & 39.0 & & & & & & \\
\hline $16-3,60-62$ & 8 & 1 & & 3 & & & & 2 & & 1 & & 58 & 54 & 4 & 88 & & & 1 & & & 220 \\
\hline $16-3,60-62$ & 3.6 & 0.5 & & 1.4 & & & & 0.9 & & 0.5 & & 26.3 & 24.5 & 1.8 & 40.0 & & & 0.5 & & & \\
\hline $16-5,60-62$ & 1 & 1 & & 8 & & & & 2 & & 1 & & 111 & 20 & & 99 & & & & & & 243 \\
\hline $16-5,60-62$ & 0.4 & 0.4 & & 3.3 & & & & 0.8 & & 0.4 & & 45.7 & 8.2 & & 40.8 & & & & & & \\
\hline $16-6,60-62$ & 2 & 1 & & 3 & & 1 & & & & 1 & & 64 & 9 & & 131 & & & & 3 & & 215 \\
\hline
\end{tabular}




\begin{tabular}{|c|c|c|c|c|c|c|c|c|c|c|c|c|c|c|c|c|c|c|c|c|c|c|c|c|c|}
\hline $\begin{array}{c}\text { Sample } \\
\text { (Interval in } \mathrm{cm} \text { ) }\end{array}$ & 苞 & 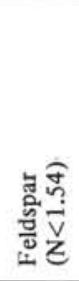 & 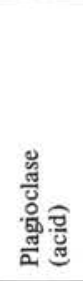 & 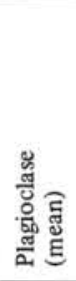 & 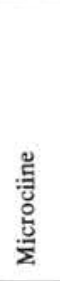 & 芯 & 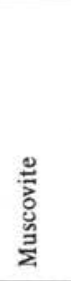 & 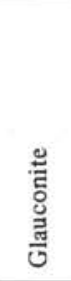 & 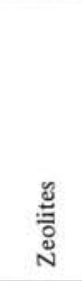 & 竞 & 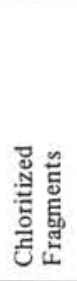 & 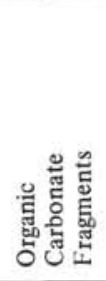 & 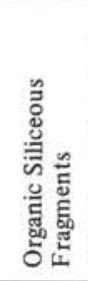 & 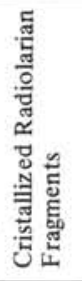 & $\frac{.}{\frac{\partial}{0}}$ & 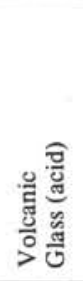 & 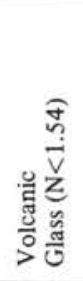 & 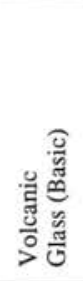 & 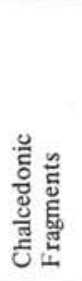 & 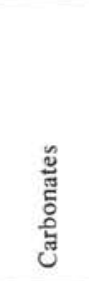 & 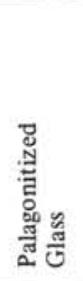 & 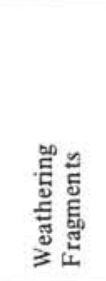 & 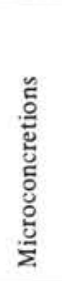 & 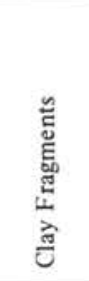 & 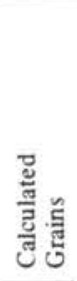 \\
\hline $15-6,60-62$ & 0.9 & 0.5 & & 1.4 & & & & & 0.5 & & & & 0.5 & & 29.7 & 4.2 & & 60.9 & & & & 1.4 & & & \\
\hline $16, \mathrm{CC}$ & 27 & 3 & & 13 & & & & & 99 & & 7 & & 3 & & & 6 & & 2.8 & & & & 45 & & 47 & 278 \\
\hline $16, \mathrm{CC}$ & 9.7 & 1.1 & & 4.7 & & & & & 35.7 & & 2.5 & & 11 & & & 2.1 & & 10.1 & & & & 16.2 & & 16.8 & \\
\hline $19-1,110-112$ & 35 & 3 & 1 & 18 & & & & & 2 & 1 & 58 & & & & & 2 & & 27 & & & & 59 & & & 206 \\
\hline $19-1,110-112$ & 17.0 & 1.5 & 0.5 & 8.8 & & & & & 1.0 & 0.5 & 28.1 & & & & & 1.0 & & 13.0 & & & & 28.6 & & & \\
\hline $19-3,75-77$ & 23 & 2 & 1 & 22 & & & & & 26 & 2 & 53 & & & & & 6 & & 10 & & & & 83 & & & 228 \\
\hline $19-3,75-77$ & 10.2 & 0.9 & 0.4 & 9.6 & & & & & 11.3 & 0.9 & 23.2 & & & & & 2.6 & & 4.4 & & & & 30.4 & & & \\
\hline $19-4,10-12$ & 10 & & & 6 & & & & & 19 & & 14 & & 1 & & & 1 & & 7 & & 85 & & 72 & & 18 & 233 \\
\hline $19-4,10-12$ & 4.3 & & & 2.6 & & & & & 8.1 & & 6.0 & & 0.4 & & & 0.4 & & 3.0 & & 36.5 & & 31.0 & & 7.7 & \\
\hline $19-6,140-142$ & 3 & 1 & 1 & 1 & & & & 1 & 9 & & & 23 & & & & 3 & & 2 & & & & 27 & & 128 & 249 \\
\hline $19-6,140-142$ & 1.2 & 0.4 & 0.4 & 0.4 & & & & 0.4 & 3.6 & & & 29.4 & & & & 1.2 & & 0.8 & & & & 10.8 & & 51.4 & \\
\hline $19, \mathrm{CC}$ & 20 & 4 & & 18 & & & & 5 & 5 & & & 7 & & & & 2 & & 27 & & & & 132 & & & 220 \\
\hline $19, \mathrm{CC}$ & 9.1 & 1.8 & & 8.2 & & & & 2.3 & 2.3 & & & 32 & & & & 0.9 & & 12.3 & & & & 59.9 & & & \\
\hline $20-1,80-82$ & 15 & 6 & 10 & 23 & 1 & & & 2 & 114 & & 50 & & & & & 1 & & 9 & & 1 & & 58 & & 18 & 308 \\
\hline $20-1,80-82$ & 4.9 & 1.9 & 32 & 7.5 & 0.3 & & & 0.6 & 37.3 & & 16.2 & & & & & 0.3 & & 2.9 & & 0.3 & & 13.8 & & 5.8 & \\
\hline $\begin{array}{l}20-2,80-82 \\
20-2,80-82\end{array}$ & 13 & 2 & 5 & 6 & & & & 1 & 3 & & 6 & & & & & 3 & & 4 & & 14 & & 19 & & 14 & 90 \\
\hline $20-4,64-66$ & 150 & 29 & 23 & 14 & & 1 & & 2 & 25 & & 17 & 1 & & & & 2 & 1 & 4 & 2 & 8 & & 8 & & 242 & 529 \\
\hline $20-4,64-66$ & 28.5 & 5.5 & 4.3 & 26 & & 0.2 & & 0.3 & 4.7 & & 3.2 & 0.2 & & & & 0.3 & 0.2 & 0.7 & 0.3 & 1.5 & & 1.5 & & 460 & \\
\hline $20-6,135-137$ & 83 & 9 & 10 & 10 & 1 & & 1 & 3 & 17 & & 54 & 38 & & & & 3 & & 4 & & & & & & 92 & 325 \\
\hline $20-6,135-137$ & 25.5 & 2.8 & 3.1 & 3.1 & 0.3 & & 0.3 & 0.9 & 5.2 & & 16.6 & 11.7 & & & & 0.9 & & 1.2 & & & & & & 28.4 & \\
\hline $20, \mathrm{CC}$ & 2 & & & 3 & & & & & 8 & & & 58 & & & & & & 8 & & & & 276 & & & 355 \\
\hline $20, \mathrm{CC}$ & 0.6 & & & 0.8 & & & & & 2.2 & & & 16.2 & & & & & & 2.2 & & & & 78.0 & & & \\
\hline $21-1,60-62$ & 9 & 3 & 2 & 5 & & & & 3 & 10 & & & & & & & & 1 & 4 & & 291 & & 13 & & & 341 \\
\hline $21-1,60-62$ & 2.6 & 0.9 & 0.6 & 1.5 & & & & 0.9 & 2.9 & & & & & & & & 0.3 & 1.2 & & 85.3 & & 3.8 & & & \\
\hline $21-3,100-104$ & 218 & 41 & 10 & 8 & 1 & & & 4 & 8 & & 18 & & & & & 2 & 1 & 1 & & 32 & & $53^{3}$ & & & 397 \\
\hline $21-3,100-104$ & 55.2 & 10.3 & 2.5 & 2.0 & 0.2 & & & 1.0 & 2.0 & & 4.5 & & & & & 0.5 & 0.2 & 0.2 & & 8.1 & & 13.3 & & & \\
\hline $21-5,60-62$ & 156 & 33 & 15 & 4 & 1 & & & 7 & 1 & & 23 & & & & & 1 & & 8 & & 9 & & & & 98 & 356 \\
\hline $21-5,60-62$ & 44.0 & 9.3 & 4.2 & 1.1 & 0.3 & & & 1.9 & 0.3 & & 6.5 & & & & & 0.3 & & 2.2 & & 2.5 & & & & 27.4 & \\
\hline 21 , CC & 40 & 15 & 5 & 1 & & & & & 1 & & & 39 & & & & 3 & & 7 & 28 & & & 28 & & 95 & 262 \\
\hline $21, \mathrm{CC}$ & 15.3 & 5.8 & 1.9 & 0.4 & & & & & 0.4 & & & 14.8 & & & & 1.1 & & 2.4 & 10.8 & & & 10.8 & & 36.3 & \\
\hline $22, \mathrm{CC}$ & 18 & 3 & 3 & 2 & & & & & & & 1 & 13 & 2 & & & 7 & & 13 & & & & & & 197 & 259 \\
\hline $22, \mathrm{CC}$ & 6.9 & 1.2 & 1.2 & 0.8 & & & & & & & 0.4 & 5.0 & 0.8 & & & 2.7 & & 5.0 & & & & & & 76.0 & \\
\hline $23-1,110-112$ & 215 & 37 & 10 & 3 & 1 & & & 53 & 2 & & $\begin{array}{l}0.4 \\
5\end{array}$ & & & & & & & 2.0 & 2 & 1 & & 25 & & & 356 \\
\hline $23-1,110-112$ & 60.8 & 10.4 & 2.8 & 0.8 & 0.2 & & & 14.9 & 0.5 & & 1.4 & & & & & & & 0.5 & 0.5 & 0.2 & & 7.0 & & & \\
\hline $23-3,77-79$ & 233 & 47 & 10 & & 2 & & & 9 & & & 5 & & & & & & & & 2 & 1.2 & & 54 & & & 363 \\
\hline $23-3,77-79$ & 64.3 & 13.0 & 2.8 & & 0.5 & & & 2.5 & & & 1.4 & & & & & & & & 0.5 & 0.2 & & 14.8 & & & \\
\hline $23-5,80-82$ & 216 & 65 & 16 & 2 & 3 & & & 9 & & & 9 & & & & & & & 1 & 1 & & & $\begin{array}{l}14.0 \\
55\end{array}$ & & & 377 \\
\hline $23-5,80-82$ & 57.5 & 17.2 & 4.2 & 0.5 & 0.8 & & & 2.4 & & & 2.4 & & & & & & & 0.2 & 0.2 & & & 14.6 & & & \\
\hline $23, \mathrm{CC}$ & 98 & 32 & 2 & & & & & & & & 26 & 5 & 1 & & & & & & 5 & & & 79 & & & 248 \\
\hline $23, \mathrm{CC}$ & 39.9 & 12.8 & 0.8 & & & & & & & & $\begin{array}{l}20 \\
10.4\end{array}$ & 2.0 & 0.4 & & & & & & 2.0 & & & 31.7 & & & \\
\hline $24-1,27-29$ & 97 & 36 & 4 & & & & 1 & & & & 10 & & & 2 & & & & & & & & 5.3 & & & 203 \\
\hline $24-1,27-29$ & 47.8 & 17.7 & 2.0 & & & & 0.5 & & & & 4.9 & & & 1.0 & & & & & & & & 26.1 & & & \\
\hline $24-2,49-51$ & 86 & 36 & 8 & 2 & & & & & & & 17.9 & & & 2 & & & & & & 4 & & 75 & & & 230 \\
\hline $24-2,49-51$ & 37.2 & 15.7 & 3.5 & 0.9 & & & & & & & 7.4 & & & 0.9 & & & & & & 1.7 & & 327 & & & \\
\hline $24-4,2-4$ & 243 & 53 & 18 & 1 & & 2 & 1 & 3 & & & $2^{1.4}$ & & & & & & & 1 & 1 & & & 65 & & & 390 \\
\hline $24-4,2-4$ & 62.5 & 13.7 & 4.6 & 0.2 & & 0.5 & 0.2 & 0.7 & & & 0.5 & & & & & & & 0.2 & 0.2 & & & $\begin{array}{l}03 \\
16.7\end{array}$ & & & \\
\hline $24-6,60-62$ & 196 & 38 & 15 & 1 & 3 & & & 7 & & & 1 & & & & & & & & 6 & & & 42 & & & 309 \\
\hline $24-6,60-62$ & 63.5 & 12.5 & 4.8 & 0.3 & 0.9 & & & 2.2 & & & 0.3 & & & & & & & & 1.9 & & & 13.6 & & & \\
\hline $24, \mathrm{CC}$ & 103 & 34 & 3 & 1 & 1 & & & & & & 5 & 4 & 1 & & & & & & & & & 80 & & & 232 \\
\hline
\end{tabular}




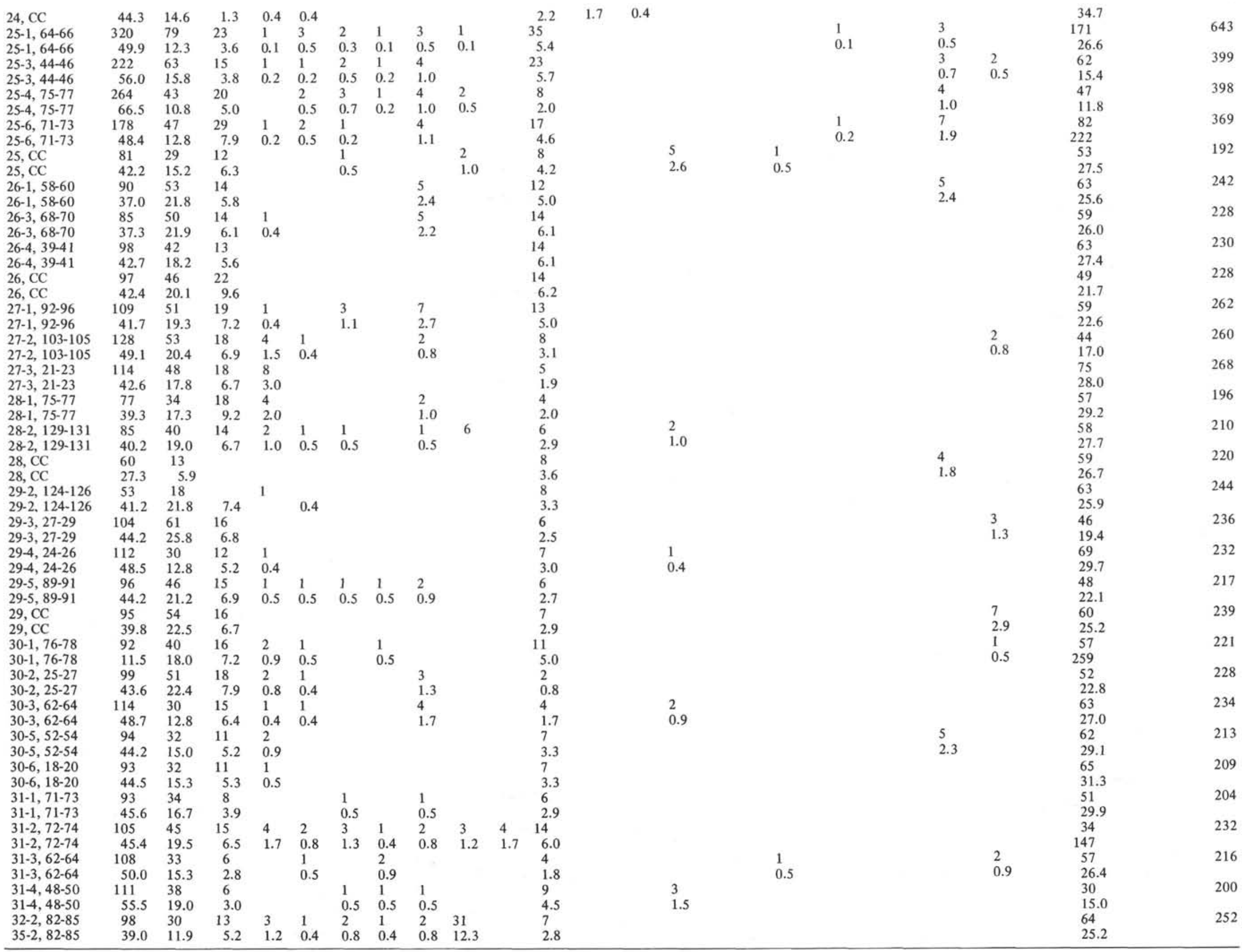


TABLE 3

Chemical Composition of Sediments from Site 348 DSDP Leg 38 from X-Ray Fluorescent Analysis

\begin{tabular}{|c|c|c|c|c|c|c|c|c|c|c|}
\hline \multirow{2}{*}{$\begin{array}{c}\text { Sample } \\
\text { (Interval in } \mathrm{cm} \text { ) }\end{array}$} & \multirow[b]{2}{*}{$\mathrm{SiO}_{2}$} & \multirow[b]{2}{*}{$\mathrm{TiO}_{2}$} & \multirow[b]{2}{*}{$\mathrm{Al}_{2} \mathrm{O}_{3}$} & \multirow[b]{2}{*}{$\mathrm{Fe}$} & \multicolumn{3}{|c|}{ Percentage } & \multirow[b]{2}{*}{$\mathrm{K}_{2} \mathrm{O}$} & \multirow[b]{2}{*}{$\mathrm{Na}_{2} \mathrm{O}$} & \multirow[b]{2}{*}{ L.O.I } \\
\hline & & & & & $\mathrm{MnO}$ & $\mathrm{MgO}$ & $\mathrm{CaO}$ & & & \\
\hline $1-3,100-104$ & 58.8 & 0.91 & 15.1 & 6.37 & 0.22 & 2.69 & 0.64 & 2.55 & 2.32 & 7.48 \\
\hline $1-5,90-92$ & 54.2 & 0.78 & 14.7 & 5.18 & 0.14 & 3.52 & 5.59 & 3.20 & 1.79 & 9.05 \\
\hline $1, \mathrm{CC}$ & 50.7 & 1.06 & 13.6 & 6.19 & 0.15 & 3.95 & 5.30 & 2.69 & 2.96 & 10.75 \\
\hline $2-1,98-100$ & 52.2 & 0.82 & 13.5 & 5.64 & 0.19 & 3.15 & 5.64 & 3.12 & & 10.05 \\
\hline $2-2,50-52$ & 45.4 & 0.99 & 12.0 & 5.76 & 0.13 & 2.76 & 10.0 & 2.78 & 2.60 & 14.10 \\
\hline $2, \mathrm{CC}$ & 54.5 & 0.91 & 14.7 & 6.96 & 0.14 & 2.75 & 3.11 & & & 9.00 \\
\hline $3-1,99-101$ & 54.7 & 1.21 & 15.1 & 7.14 & 0.10 & 2.87 & 1.02 & 3.24 & 2.23 & 8.83 \\
\hline $3-4,99-101$ & 56.2 & 1.08 & 13.9 & 6.17 & 0.53 & 2.90 & 1.60 & 3.26 & 2.12 & 8.55 \\
\hline $3, \mathrm{CC}$ & 56.5 & 1.20 & 14.8 & 7.63 & 0.12 & 2.97 & 1.14 & 3.07 & & 8.20 \\
\hline $4-1,119-121$ & 57.1 & 1.05 & 14.8 & 6.73 & 0.13 & 2.56 & 1.02 & 5 & & 8.09 \\
\hline $5-1,99-100$ & 54.4 & 1.1 & 15.1 & 6.51 & 0.11 & 1.90 & 1.10 & & & 7.75 \\
\hline $6-5,73-75$ & 37.4 & 0.52 & 8.45 & 3.04 & 0.28 & 2.28 & 20.3 & 0.67 & 3. & 21.68 \\
\hline $6, \mathrm{CC}$ & 47.6 & 1.60 & 10.0 & 7.45 & 0.16 & 3.00 & 10.6 & 1.43 & 3.3 & 11.94 \\
\hline $8-2,77-79$ & 53.5 & 1.06 & 13.7 & 6.67 & 0.08 & 2.97 & 1.91 & 2.6 & & 11.16 \\
\hline $8-3,75-77$ & 53.7 & 1.25 & 12.5 & 7.53 & 0.08 & 2.22 & 2.02 & 62 & & 11.34 \\
\hline $9-1,138-140$ & 48. & 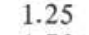 & 12 & 8.76 & 0.10 & 3.40 & 2.01 & & 3. & 13.84 \\
\hline $11-1,80-81$ & 52.2 & 1.70 & 11.5 & 7.90 & 0.11 & 2.48 & 3.68 & 1.52 & 3.63 & 12.01 \\
\hline $12-2,60-62$ & 54.5 & 1.80 & 9.55 & 7.95 & 0.11 & 3.02 & 4.05 & 1.33 & 3.50 & 10.85 \\
\hline $12-4,20-22$ & 52.6 & 1.78 & 10.7 & 8.79 & 0.11 & 2.38 & 3.87 & 1.48 & & 12.25 \\
\hline $14-2,20-22$ & 52.6 & 1.48 & 10.7 & 8.19 & 0.12 & 2.62 & 3.8 & 1.62 & 3.50 & 11.70 \\
\hline $14-6,20-22$ & 51.7 & 1.23 & 10.3 & 8.40 & 0.12 & 4.18 & 4.3 & & & 11.17 \\
\hline $14, \mathrm{CC}$ & 53 & 1.3 & 10.4 & 10.8 & 0.15 & 3.10 & 6.3 & 0. & 9 & 6.33 \\
\hline $15-1,60-62$ & 54.4 & 1.15 & 11.7 & 6.41 & 0.07 & 1.97 & 2.78 & 1.89 & 3.54 & 13.17 \\
\hline $15-2,60-62$ & 54.8 & 1.08 & 11.1 & 5.13 & 0.05 & 3.89 & 2.54 & 1.8 & & 13.45 \\
\hline $15, \mathrm{CC}$ & 55. & 1.36 & 11.6 & 8.78 & 0.10 & 2.23 & 5.07 & 1.59 & & 6.29 \\
\hline $16-1,60-62$ & 56.9 & 1.06 & 11.8 & $6 .($ & 0.01 & 1.31 & & & & 11.34 \\
\hline $16-6,60-62$ & 56 & 1.36 & & 7.56 & 0.13 & 2.38 & 4.38 & .18 & 5 & 10.02 \\
\hline $16, \mathrm{CC}$ & 55.2 & 1.20 & 11.4 & 7.10 & 0.03 & 3.41 & 1.90 & 1.98 & 2.5 & 12.28 \\
\hline $19-1,110-112$ & 53.5 & 1.56 & 11.0 & 7.70 & 0.06 & 5.35 & 2.06 & 1.91 & 2.54 & 11.41 \\
\hline $19-3,75-77$ & 48.7 & 1.81 & 10. & 9.10 & 0.07 & 5.63 & 2.48 & 1.22 & & 13.70 \\
\hline $19-4,10-12$ & 51.0 & 1.39 & 10.7 & 8.06 & 0.08 & 5.04 & 2.1 & 1.67 & 30 & 13.40 \\
\hline $19-6,140-142$ & 46.0 & 1.64 & 9.67 & 8.54 & 0.25 & 7.72 & 4.8 & 0.8 & & 14.15 \\
\hline $19, \mathrm{CC}$ & 47 & 1.48 & 10.4 & 12.4 & 0.09 & 5.13 & 2.5 & 0.92 & & 11.25 \\
\hline $20-1,80-82$ & 46.0 & 1.77 & 9.40 & 10.1 & 0.20 & 6.27 & 4.05 & 0.72 & 2. & 14.36 \\
\hline $20-2,80-82$ & 47.6 & 1.69 & 10.2 & 9.80 & 0.08 & 6.75 & 2.33 & 1.15 & 2. & 13.91 \\
\hline $20-6,135-137$ & 40. & 1.62 & 7.80 & 8.40 & 0.38 & 5.76 & 11.3 & 0.83 & 2. & 17.20 \\
\hline $20, \mathrm{CC}$ & 46.7 & 2.26 & 9.17 & 11.5 & 0.17 & 4.92 & 4.68 & 0.84 & 2.3 & 12.76 \\
\hline $21-1,60-62$ & & 1.42 & 10.6 & & 0.06 & & & .41 & 2.47 & 13.58 \\
\hline $21-3,100-104$ & 51.8 & 0.9 & 12.4 & & 0.05 & 5.20 & 1.6 & 2.21 & & 13.14 \\
\hline $21-5,60-62$ & 54.4 & 1.07 & 11.7 & .23 & 0.03 & 5.27 & 1.44 & 2.62 & 2. & 12.40 \\
\hline $22, \mathrm{CC}$ & 51.8 & 1.36 & 12.9 & 3.74 & 0.07 & 3.64 & 1.71 & 2.2 & 1. & 12.29 \\
\hline $23-1,110-112$ & 52. & 1.53 & 13.3 & 7.40 & 0.06 & 3.64 & 1.11 & 2.38 & & 12.20 \\
\hline & 55 & 1.02 & & & 0.04 & & & & & \\
\hline $23-5,80-82$ & 54. & 1.5 & 14 & & 0.04 & 2.3 & 1.5 & 2.49 & 4 & 11.03 \\
\hline $23, \mathrm{CC}$ & 58.3 & 1.37 & 14.0 & 6.60 & 0.04 & 2.72 & 1.38 & 2.35 & 1.3 & 9.13 \\
\hline $24-1,27-29$ & 56.0 & 1.68 & 14.2 & 4.73 & 0.05 & 3.20 & 1.3 & 2.21 & $2 .($ & 12.63 \\
\hline $24-2,49-51$ & 54.9 & 1.33 & 14.1 & & 0.05 & 2.17 & & & & 12.34 \\
\hline $24-6$ & 56 & & & & 0.04 & & & & & \\
\hline $24-6,60-62$ & 54.2 & 1.49 & 14.95 & 6.23 & 0.04 & 3.71 & 1.24 & 2.26 & 1. & 11.83 \\
\hline $24, \mathrm{CC}$ & 55.0 & 1.55 & 14.8 & 7.48 & 0.05 & 2.66 & 1.47 & 2.62 & 1.35 & 9.53 \\
\hline $25-1,64-66$ & 55.6 & 1.42 & 14.8 & 6.42 & 0.05 & 2.82 & 1.19 & 2.48 & 1. & 10.87 \\
\hline $25-3,44-46$ & 55.2 & 1.41 & 14.0 & 6.87 & 0.04 & 2.85 & 1.56 & 2.20 & 1. & 11.59 \\
\hline $25-4,75-77$ & 54.6 & 1.43 & 14.4 & 7.27 & 0.04 & 2.68 & & 2.50 & & 11.46 \\
\hline $25-6,71-78$ & 56.5 & 1.5 & 14.5 & & 0.06 & 2.78 & 1.43 & 2.34 & & 11.31 \\
\hline $25, \mathrm{CC}$ & 56.1 & 1.45 & 13.9 & 7.59 & 0.02 & 2.87 & 1.55 & 2.47 & 1.33 & 9.29 \\
\hline $26-1,58-60$ & 55.6 & 1.36 & 13.9 & 6.88 & 0.05 & 2.66 & 1.42 & 2.11 & 2.23 & 10.84 \\
\hline $26-3,68-70$ & 56.8 & 1.44 & 14.4 & 6.67 & 0.05 & 2.13 & 1.56 & 2.14 & 1.75 & 9.66 \\
\hline $26-4,39-41$ & 56.8 & 1.52 & 14.2 & 7.20 & 0.04 & 1.80 & 1.53 & 2.47 & 1.32 & 10.18 \\
\hline $26, \mathrm{CC}$ & 58.5 & 1.39 & 13.5 & 7.20 & 0.02 & 2.26 & 1.83 & 2.42 & 1.40 & 8.04 \\
\hline $27-1,92-96$ & 57.5 & 1.20 & 14.4 & 6.08 & 0.04 & 2.07 & 1.45 & 2.39 & 1.39 & 10.53 \\
\hline $27-2,103-105$ & 56.4 & 1.35 & 15.1 & 6.52 & 0.04 & 2.54 & 1.49 & 2.45 & 1.04 & 10.50 \\
\hline $27-3,21-23$ & 58.2 & 1.32 & 14.9 & 6.17 & 0.04 & 2.34 & 1.42 & 2.15 & 1.48 & 10.20 \\
\hline $28-1,75-77$ & 56.5 & 1.49 & 14.5 & 6.71 & 0.05 & 1.55 & 1.40 & 2.47 & 1.52 & 10.44 \\
\hline $28-2,129-131$ & 54.6 & 1.27 & 14.3 & 7.90 & 0.04 & 2.13 & 1.62 & 2.25 & 1.73 & 10.76 \\
\hline $28, \mathrm{CC}$ & 59.8 & 1.34 & 14.8 & 6.99 & 0.02 & 1.34 & 1.43 & 2.42 & 1.29 & 7.81 \\
\hline
\end{tabular}


TABLE 3 - Continued

\begin{tabular}{|c|c|c|c|c|c|c|c|c|c|c|}
\hline \multirow{2}{*}{$\begin{array}{c}\text { Sample } \\
\text { (Interval in } \mathrm{cm} \text { ) }\end{array}$} & \multirow[b]{2}{*}{$\mathrm{SiO}_{2}$} & \multicolumn{9}{|c|}{ Percentage } \\
\hline & & $\mathrm{TiO}_{2}$ & $\mathrm{Al}_{2} \mathrm{O}_{3}$ & $\mathrm{Fe}$ & $\mathrm{MnO}$ & $\mathrm{MgO}$ & $\mathrm{CaO}$ & $\mathrm{K}_{2} \mathrm{O}$ & $\mathrm{Na}_{2} \mathrm{O}$ & L.O.I. \\
\hline $29-2$ & 57.9 & 1.33 & 14.85 & 5.73 & 0.04 & 2.77 & 1.39 & 2.37 & & 10.41 \\
\hline $29-4,24-26$ & 60.5 & 1.54 & 13.8 & 6.64 & 0.04 & 0.70 & 1.47 & 2.23 & 1.57 & 9.29 \\
\hline $29, \mathrm{CC}$ & 61.6 & 1.28 & 13.7 & 6.18 & 0.04 & 2.00 & 1.27 & 2.13 & 1.23 & 7.16 \\
\hline $30-1,76-78$ & 52.6 & 1.56 & 15.7 & 7.48 & 0.02 & 2.31 & 1.55 & 2.17 & 1.56 & 12.18 \\
\hline $30-2,25-27$ & 64.8 & 1.46 & 13.3 & 3.27 & 0.05 & 2.49 & 1.44 & 1.96 & 1.51 & 8.22 \\
\hline $30-3,62-64$ & 57.2 & 1.32 & 13.1 & 7.33 & 0.04 & 2.39 & 1.42 & 2.12 & 1.57 & 10.12 \\
\hline $30-5,52-54$ & 57.2 & 1.51 & 15.0 & 6.47 & 0.05 & 2.00 & 1.82 & 2.22 & & 9.71 \\
\hline $30-6,18-20$ & 62.3 & 1.38 & 13.8 & 3.94 & 0.05 & 2.86 & 1.46 & 2.10 & 1.52 & 9.02 \\
\hline $31-1,71-73$ & 58.0 & 1.38 & 14.9 & 6.30 & 0.04 & 2.67 & 1.30 & 2.45 & 1.38 & 8.97 \\
\hline $31-2,72-74$ & 57.1 & 1.55 & 15.8 & 6.12 & 0.05 & 2.71 & 1.42 & 2.72 & 1.41 & 8.88 \\
\hline $31-3,61-64$ & 46.6 & 2.24 & 19.1 & 7.90 & 0.07 & 2.76 & 1.65 & 1.92 & 2.54 & 12.06 \\
\hline $31-4,48-50$ & 55.7 & 1.62 & 17.0 & 5.54 & 0.03 & 3.54 & 1.42 & 2.68 & 0.77 & 8.86 \\
\hline $32-2,82-85$ & 59.5 & 1.61 & 15.2 & 6.06 & 0.04 & 2.94 & 1.32 & 2.58 & 1.21 & 7.00 \\
\hline
\end{tabular}

mixed-layered clays, mainly of the hydromica-montmorillonitic composition. These components are probably terrigenous, an indication of a gradual increase in the supply of erosional material in the Pliocene.

\section{Unit 1}

A comparison of the Unit 1 (Pleistocene) deposits with the sediments of Unit 2 indicates many differences. There is a considerable decrease of biogenic material expressed chiefly by a sharp decrease in the content of amorphous silica and calcium carbonate. The content of these components is more characteristic for that indicated in Unit 3.

Analysis of the composition of the coarse silt fraction of Unit 1 shows a mixing of terrigenous and volcanogenic components with some predominance of the volcanogenic. The terrigenous composition of this fraction (quartz, potassium feldspars, hornblende, weathered minerals) is very similar to the composition

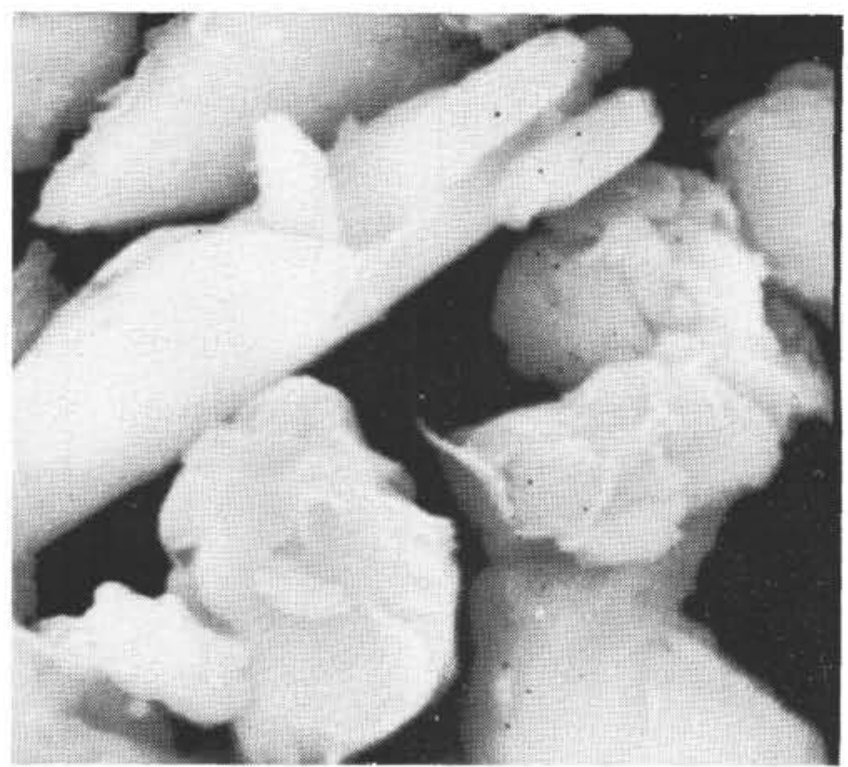

Figure 1. Less than $5 \mu \mathrm{m}$ fraction of the sediments from $a$ depth of 525-526.5 meters. Core 32. $\times 9500$ magnification. of the silts of lower units (Table 1). Volcanogenic material (devitrified glass and palagonitized glass) is more significant in the silts of Unit 1 than in Unit 3. This is probably caused by a smaller degree of alteration by diagenesis.

An analysis of the silt composition also indicates a sharp decrease in iron sulfide as compared with lower units. There is also an extremely low content of glauconite and iron hydroxides. Only individual grains of zeolite are present, which indicates a low degree of diagenesis of the Pleistocene sediments; this also correlates with the lower content of organic material (less than $0.1 \%$ ) in these sediments.

A similar composition is characteristic for the (less than $1 \mu \mathrm{m}$ ) fraction of Unit 1 . The mineral assemblage is very rich, and many of the minerals are well crystallized. The predominance of hydromica and the presence of considerable amounts of kaolinite and mixed-layered varieties of the hydromica-chlorite and montmorillonite points to a significant contribution by terrigenous components. However, montmorillonite, which is considered to be authigenic and formed from volcanogenic material, is also abundant. This is the second major component after hydromica in the clay fraction.

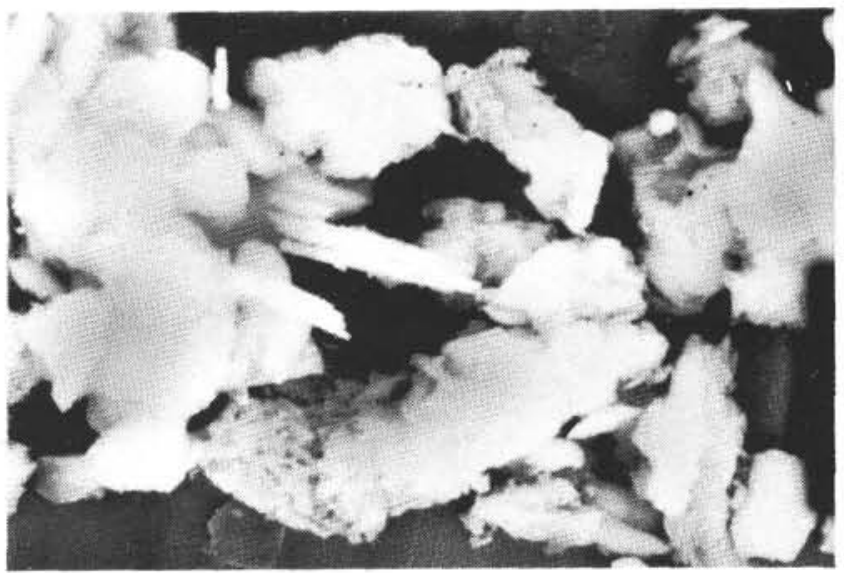

Figure 2. Less than $5 \mu \mathrm{m}$ fraction of sediments from a depth of 278-279.5 meters. Core 20. $\times 2490$ magnification. 
TABLE 4

Grain-Size Distribution of the Sediments from Site 348 Leg 38

\begin{tabular}{|c|c|c|c|c|c|c|c|c|c|c|}
\hline Sample & Weight & Wetness & & & & & & & Fracti & ons (\%) \\
\hline (Interval in $\mathrm{cm}$ ) & of Samples & $(\%)$ & $7-5$ & $5-3$ & $3-2$ & $2-1$ & $1-0.5$ & $0.5-0.25$ & $0.25-0.1$ & $0.1-0.05$ \\
\hline $1-1,70-72$ & 4.57 & 60.2 & & & & & 1.97 & 2.19 & 24.07 & 3.28 \\
\hline $1-3,100-104$ & 13.25 & 62.4 & & & & & 0.45 & 0.68 & 4.15 & 6.04 \\
\hline $1-4,100-102$ & 4.81 & 55.4 & & & & & 0.62 & 0.62 & 1.56 & 3.12 \\
\hline $1-5,90-92$ & 7.58 & 35.2 & & & & & & & 4.62 & 9.76 \\
\hline $1, \mathrm{CC}$ & 18.55 & 57.2 & & & & & 0.49 & 0.32 & 2.43 & 2.10 \\
\hline $2-1,98-100$ & 5.10 & 39.1 & & & & & & 0.59 & 5.69 & 2.94 \\
\hline $2-2,50-52$ & 6.33 & 51.1 & & & & & & 0.79 & 6.16 & 4.58 \\
\hline $2-4,99-101$ & 6.15 & 42.7 & & & & & & & 2.28 & 0.65 \\
\hline $2, \mathrm{CC}$ & 11.60 & 40.2 & & & & & 1.03 & 0.86 & 4.66 & 4.74 \\
\hline $3-1,99-101$ & 8.03 & 42.6 & & & & & & & 1.74 & 1.87 \\
\hline $3-3,99-101$ & 9.93 & 49.8 & & & & & & & 1.21 & 1.41 \\
\hline $3-4,99-101$ & 8.43 & 39.4 & & & & & & 0.47 & 3.44 & 13.00 \\
\hline $3-5,89-91$ & 11.25 & 55.4 & & & & & & & 0.44 & 10.44 \\
\hline $3, \mathrm{CC}$ & 11.00 & 47.6 & & & & & & 0.36 & 3.18 & 5.91 \\
\hline $4-1,119-121$ & 11.43 & 51.8 & & & & & & & 1.22 & 1.22 \\
\hline $4-2,99-101$ & 8.26 & 60.0 & & & & & & & 1.09 & 1.21 \\
\hline $5-1,99-101$ & 12.26 & 44.0 & & & & & & & 0.33 & 2.37 \\
\hline $5-2,130-144$ & 13.58 & 69.4 & & & & & & & 0.74 & 1.25 \\
\hline $5, \mathrm{CC}$ & 6.93 & 56.8 & & & & & 5.34 & 0.58 & 3.61 & 5.34 \\
\hline $6-1,139-141$ & 6.61 & 67.6 & & & & & & & 0.61 & 0.76 \\
\hline $6-2,29-31$ & 12.12 & 66.0 & & & & & & & 0.41 & 0.74 \\
\hline $6-4,84-86$ & 13.57 & 60.1 & & & & & & & 0.37 & 0.37 \\
\hline $6-5,73-75$ & 10.67 & 50.6 & & & & & & & 0.37 & 0.48 \\
\hline $6, \mathrm{CC}$ & 19.02 & 55.2 & & & & & & 0.05 & 1.26 & 4.10 \\
\hline $7-1,74-76$ & 13.11 & 66.8 & & & & & & & 0.84 & 0.38 \\
\hline $7-3,74-76$ & 8.20 & 62.3 & & & & & & & 0.49 & 0.98 \\
\hline $7-4,75-77$ & 8.95 & 67.0 & & & & & & & 0.22 & 0.44 \\
\hline $7, \mathrm{CC}$ & 18.46 & 52.7 & & & & & & & 1.30 & 1.63 \\
\hline $8-2,75-77$ & 9.37 & 57.6 & & & & & & & 0.43 & 0.53 \\
\hline $8-3 ; 75-77$ & 8.81 & 46.4 & & & & & & & 0.23 & 0.34 \\
\hline $9-1,138-140$ & 7.36 & 68.3 & & & & & & & 0.41 & 0.68 \\
\hline $9-2,75-77$ & 9.53 & 65.6 & & & & & & & 0.52 & 0.73 \\
\hline $9-5,75-77$ & 12.18 & 62.9 & & & & & & & 0.82 & 1.31 \\
\hline $9, \mathrm{CC}$ & 11.24 & 42.5 & & & & & & & 1.33 & 1.96 \\
\hline $11-1,80-82$ & 6.06 & 64.3 & & & & & & & 0.99 & 1.65 \\
\hline $11-2,80-81$ & 11.43 & 62.7 & & & & & & & 1.75 & 2.01 \\
\hline $11-4,70-72$ & 9.15 & 56.2 & & & & & & & 3.28 & 4.92 \\
\hline $12-1,85-86$ & 6.38 & 60.5 & & & & & & & 0.78 & 1.88 \\
\hline $12-2,60-62$ & 9.76 & 60.3 & & & & & & & 2.97 & 2.56 \\
\hline $12-4,20-22$ & 8.00 & 65.0 & & & & & & & 2.88 & 1.88 \\
\hline $13-1,80-82$ & 5.71 & 21.5 & & & & & & & 5.60 & 1.75 \\
\hline $13-2,20-22$ & 8.35 & 66.9 & & & & & & & 0.60 & 1.80 \\
\hline $13, \mathrm{CC}$ & 8.78 & 56.4 & & & & & & 0.57 & 11.96 & 15.48 \\
\hline $14-1,20-22$ & 7.36 & 62.1 & & & & & & & 3.67 & 1.63 \\
\hline $14-2,20-22$ & 7.08 & 37.4 & & & & & & & 2.82 & 2.68 \\
\hline $14-4,20-22$ & 9.17 & 55.8 & & & & & & & 0.98 & 0.76 \\
\hline $14-5,20-22$ & 8.53 & 61.3 & & & & & & & 0.59 & 0.59 \\
\hline $14-6,20-22$ & 8.19 & 58.0 & & & & & & & 2.93 & 1.71 \\
\hline $14, \mathrm{CC}$ & 10.46 & 47.4 & & & & & 6.02 & 8.03 & 18.55 & 10.61 \\
\hline $15-1,60-62$ & 6.75 & 61.1 & & & & & & & 0.74 & 0.59 \\
\hline $15-2,60-62$ & 6.93 & 61.8 & & & & & & & 0.58 & 0.58 \\
\hline $15, \mathrm{CC}$ & 7.87 & 54.5 & & & & & 0.25 & 0.89 & 13.34 & 10.17 \\
\hline $16-1,60-62$ & 7.19 & 55.0 & & & & & & & 2.64 & 0.97 \\
\hline $16-3,60-62$ & 7.89 & 62.2 & & & & & & & 1.40 & 0.64 \\
\hline $16-5,60-62$ & 7.81 & 59.6 & & & & & & & 3.07 & 1.41 \\
\hline $16-6,60-62$ & 7.42 & 53.6 & & & & & & & 4.45 & 1.21 \\
\hline $16, \mathrm{CC}$ & 10.38 & 48.1 & & & & & & & 1.16 & 1.45 \\
\hline $19-1,110-112$ & 8.40 & 50.4 & & & & & & & 1.19 & 0.24 \\
\hline $19-3,75-77$ & 5.73 & 55.9 & & & & & & & 0.87 & 0.35 \\
\hline $19-4,10-12$ & 7.80 & 53.3 & & & & & & & 2.44 & 0.26 \\
\hline $19-6,140-142$ & 8.20 & 44.3 & & 9.02 & 2.93 & 2.93 & 1.83 & 1.71 & 1.95 & 0.12 \\
\hline $19, \mathrm{CC}$ & 11.87 & 43.2 & 40.49 & 18.01 & 14.23 & 6.31 & 3.79 & 3.79 & 4.12 & 0.17 \\
\hline $20-1,80-82$ & 6.97 & 50.0 & & & & & 2.15 & 1.72 & 3.44 & 0.29 \\
\hline $20-2,80-82$ & 6.85 & 47.1 & & & & & & & 3.65 & 0.15 \\
\hline $20-4,64-6$ & & 58.3 & & & & & & & 0.21 & 0.21 \\
\hline $20-6,135-137$ & 6.95 & 46.4 & 4.60 & 7.19 & 5.04 & 5.18 & 3.45 & 4.75 & 5.04 & 0.29 \\
\hline $20, C C$ & 11.05 & 42.4 & & 9.14 & 7.42 & 9.23 & 7.15 & 9.86 & 10.77 & 3.62 \\
\hline $21-1,60-62$ & 6.37 & 55.6 & & & & & & & 0.78 & 0.16 \\
\hline $21-3,100-104$ & 9.09 & 54,0 & & & & & & & 0.44 & 0.10 \\
\hline $21-5,60-62$ & 7.03 & 52.9 & & & & & & & 0.71 & 0.14 \\
\hline $21, \mathrm{CC}$ & 8.75 & 54.6 & 56.03 & 8.80 & 6.29 & 6.97 & 4.46 & 4.34 & 5.49 & 1.14 \\
\hline $22, \mathrm{CC}$ & 8.34 & 56.9 & & & & & & & 0.24 & 0.24 \\
\hline $23-1,110-112$ & 7.03 & 52.4 & & & & & & & 1.56 & 0.28 \\
\hline $23-3,77-79$ & 6.37 & 41.3 & & & & & & & 2.20 & 1.57 \\
\hline $23-5,80-82$ & 5.29 & 37.0 & & & & & & & 1.13 & 1.51 \\
\hline $23, \mathrm{CC}$ & 13.57 & 34.1 & & & & & & 1.77 & 3.32 & 5.53 \\
\hline $24-1,27-29$ & 10.29 & 37.9 & & & & & & 0.24 & 1.85 & 0.87 \\
\hline $24-2,49-51$ & 4.34 & 34.8 & & & & & & & 1.61 & 2.30 \\
\hline $24-4,204$ & 12.59 & 34.2 & & & & & & & 3.57 & 1.91 \\
\hline $24-6,60-62$ & 11.33 & 30.5 & & & & & & & 5.47 & 2.21 \\
\hline $24, \mathrm{CC}$ & 13.51 & 31.4 & & & & & & 0.15 & 2.74 & 1.11 \\
\hline $25-1,64-66$ & 11.26 & 33.9 & & & & & & & 2.84 & 2.58 \\
\hline $25-3,44-46$ & 13.52 & 32.6 & & & & & & & 2.51 & 1.11 \\
\hline $25-4,75-77$ & 7.85 & 33.2 & & & & & & & 1.15 & 1.78 \\
\hline $25-6,71-73$ & 11.11 & 32.9 & & & & & & & 0.81 & 0.90 \\
\hline $25, \mathrm{CC}$ & 14.88 & 27.3 & & & & & & 0.34 & 3.02 & 2.35 \\
\hline
\end{tabular}


TABLE 4 - Continued

\begin{tabular}{|c|c|c|c|c|c|c|c|c|c|c|c|c|c|c|}
\hline \multirow{2}{*}{$\begin{array}{c}\text { Sample } \\
\text { (Interval in } \mathrm{cm} \text { ) }\end{array}$} & \multirow{2}{*}{$\begin{array}{l}\text { Weight } \\
\text { of samples }\end{array}$} & \multirow{2}{*}{$\begin{array}{l}\text { Wetness } \\
(\%)\end{array}$} & \multicolumn{12}{|c|}{ Fractions (\%) } \\
\hline & & & $7-5$ & $5-3$ & $3-2$ & $2-1$ & $1-0.5$ & $0.5-0.25$ & $0.25-0.1$ & $0.1-0.05$ & $0.05-0.01$ & $0.01-0.005$ & $0.005-0.001$ & $<0.001$ \\
\hline $26-1,58-60$ & 5.84 & 18.0 & & & & & & & 2.57 & 3.25 & 0.86 & 12.15 & 25.86 & 55.31 \\
\hline $26-3,68-70$ & 6.03 & 28.2 & & & & & & & 2.99 & 1.99 & 0.83 & 18.74 & 25.04 & 50.41 \\
\hline $26-4,39-41$ & 11.11 & 28.5 & & & & & & & 3.15 & 1.71 & 0.36 & 21.34 & 24.48 & 48.96 \\
\hline $26, \mathrm{CC}$ & 14.93 & 28.9 & & & & & & 1.00 & 5.69 & 4.02 & 0.33 & 26.53 & 25.32 & 37.11 \\
\hline $27-1,92-96$ & 14.29 & 34.2 & & & & & & & 5.87 & 4.48 & 0.28 & 17.29 & 23.79 & 48.29 \\
\hline $27-2,103-105$ & 12.17 & 29.7 & & & & & & & 0.41 & 3.04 & 0.16 & 19.97 & 31.06 & 45.36 \\
\hline $27-3,21-23$ & 9.32 & 33.5 & & & & & & & 2.04 & 2.04 & 0.54 & 19.31 & 20.28 & 55.79 \\
\hline $28-1,75-77$ & 12.11 & 27.6 & & & & & & & 2.06 & 1.57 & 0.74 & 27.33 & 23.38 & 44.92 \\
\hline $28-2,129-131$ & 10.41 & 31.2 & & & & & & & 6.15 & 2.69 & 1.63 & 10.47 & 25.46 & 53.60 \\
\hline $28, \mathrm{CC}$ & 16.28 & 18.8 & & & & & 5.90 & 3.81 & 11.36 & 6.39 & 0.84 & 10.78 & 24.92 & 36.00 \\
\hline $29-2,124-126$ & 11.29 & 31.6 & & & & & & & 3.72 & 2.75 & 0.80 & 21.25 & 23.47 & 48.01 \\
\hline $29-3,25-27$ & 10.69 & 28.2 & & & & & & & 4.77 & 4.12 & 0.47 & 18.05 & 21.23 & 51.36 \\
\hline $29-4,24-26$ & 8.97 & 30.4 & & & & & & & 6.02 & 2.45 & 1.11 & 18.40 & 23.97 & 48.05 \\
\hline $29-5,89-91$ & 11.08 & 63.4 & & & & & & & 2.26 & 1.90 & 0.45 & 22.82 & 23.92 & 48.65 \\
\hline $29, \mathrm{CC}$ & 15.69 & 21.9 & 18.93 & 3.82 & 0.64 & 0.64 & 0.32 & 3.12 & 8.86 & 5.42 & 0.32 & 10.19 & 15.81 & 31.93 \\
\hline $30-1,76-68$ & 9.68 & 31.2 & & & & & 2.48 & 4.65 & 11.47 & 2.38 & 0.41 & 23.35 & 17.56 & 37.70 \\
\hline $30-2,25-27$ & 10.15 & 23.5 & & & & & & & 7.00 & 5.52 & 0.89 & 13.98 & 18.62 & 53.99 \\
\hline $30-3,62-64$ & 6.29 & 24.5 & & & & & & & 1.10 & 4.78 & 0.79 & 22.74 & 24.01 & 46.58 \\
\hline $30-5,52-54$ & 7.44 & 13.4 & & & & & & & 5.38 & 6.05 & 1.34 & 19.62 & 22.85 & 44.76 \\
\hline $30-6,18-20$ & 12.05 & 35.1 & & & & & & & 6.14 & 2.49 & 0.83 & 23.22 & 23.57 & 43.75 \\
\hline $31-1,71-73$ & 9.81 & 21.5 & & & & & & & 7.54 & 3.36 & 0.92 & 7.65 & 27.01 & 53.52 \\
\hline $31-2,72-74$ & 10.22 & 24.4 & & & & & 0.88 & 1.86 & 5.28 & 3.91 & 1.96 & 17.91 & 23.09 & 45.11 \\
\hline $31-3,62-64$ & 14.50 & 24.2 & & & & & & & 1.10 & 0.55 & 0.34 & 31.25 & 26.07 & 40.69 \\
\hline $31-4,48-50$ & 12.68 & 19.0 & & & & & & & 6.62 & 4.26 & 0.79 & 12.46 & 29.81 & 46.06 \\
\hline $32-2,82-85$ & 10.92 & 20.3 & & & & 2.20 & 0.55 & 3.11 & 8.79 & 10.53 & 0.64 & 11.54 & 19.05 & 43.59 \\
\hline
\end{tabular}

TABLE 5

$\mathrm{Fe}_{2} \mathrm{O}_{3}, \mathrm{TiO}_{2}$, and $\mathrm{MnO}$ Content in Sediments from Site 348, DSDP, Leg 38

\begin{tabular}{cccccc}
\hline Core & Section & $\begin{array}{c}\text { Interval } \\
(\mathrm{cm})\end{array}$ & $\mathrm{Fe}_{2} \mathrm{O}_{3}(\%)$ & $\mathrm{TiO}_{2}(\%)$ & $\mathrm{MnO}(\%)$ \\
\hline 5 & 2 & $139-141$ & 7.87 & 0.96 & 0.06 \\
7 & 3 & $74-76$ & 9.60 & 1.56 & 0.09 \\
7 & 4 & $75-77$ & 9.71 & 1.38 & 0.09 \\
7 & $\mathrm{CC}$ & & 8.59 & 1.18 & 0.06 \\
11 & 4 & $70-72$ & 11.04 & 1.86 & 0.10 \\
13 & 1 & $80-82$ & 9.31 & 1.36 & 0.09 \\
13 & $\mathrm{CC}$ & & 11.36 & 1.52 & 0.11 \\
14 & 5 & $20-27$ & 7.26 & 0.86 & 0.07 \\
16 & 5 & $60-62$ & 7.05 & 0.96 & 0.06 \\
20 & 4 & $64-66$ & 8.88 & 1.10 & 0.04 \\
21 & $\mathrm{CC}$ & & 12.26 & 1.34 & 0.05 \\
29 & 3 & $25-27$ & 7.32 & 1.16 & 0.06 \\
\hline
\end{tabular}

\section{CONCLUSIONS}

The origin of the late Cenozoic sediments in the Icelandic Basin represents a series of sedimentation stages characteristic for the North Atlantic. The contact between the sediments and underlying basalts is sedimentary. The approximately 20 -meter-thick layer of Oligocene(?) sediments, which overlies the basalts, has been formed, to a considerable degree, as a result of submarine weathering of these variolitic basalts, and/or erosion from adjacent uplifted sections of the oceanic floor. This is further supported by the presence of basaltic gravels in the lowermost layers. The high degree of zeolitization indicates the importance of the alteration products from the basalts. Zeolites of the analcite group may also be present.

The accumulation of the late Oligocene-early Miocene deposits occurred concurrently with effusive surface and submarine volcanism. The supply of the volcanogenic ash material increases upward in the section, attaining a maximum at the end of early Miocene, particularly at the boundary with middle Miocene.
Probably, the ash material consisted almost entirely of basic glass. Subsequently, this glass was altered to montmorillonite and zeolite.

The supply of the terrigenous material was very low, probably, lower than in the Pleistocene and Holocene. The factor affecting the supply of terrigenous material was probably glaciation, but may have been less operative at this time. The similarity of the Oligoceneearly Miocene deposits with contemporary sediments is displayed by the low content of biogenic components which may be indicative of a severe climate similar to the present.

The latter comment seems contradictory because the sediments of the lower stratigraphic horizon are largely enriched in organic matter. However, the level of this "enrichment" is significantly low, and it can be detected only by comparison with the "poorer" organic carbon content in the sediments of the overlying units (1 and 2). The relatively higher content of organic matter in the sediments of Unit 3, as well as the evidence of iron sulfidization, evidently points to a somewhat higher content of hydrogen sulfide in benthonic depths of the basin at that time. If this is the case, there are reasons to suggest the existence of a relatively low content of free oxygen in bottom waters. These would be associated with a slow circulation of water masses in the basin and existence of a chalistatic zone in this water. Another factor leading to stagnant water masses of the basin in the Oligocene-early Miocene times may be the steep topography of the peripheres (i.e., the Jan-Mayen Ridge system).

These conditions were favorable for the preservation of the organic matter which appeared to change its composition mainly by diagenesis. At the same time, it should be added that these conditions stimulated the dissolution of carbonate skeletons, and to some degree, the silica varieties, both of which are unstable in an alkaline environment.

Sedimentation in the middle and upper Miocene and in Pliocene (Unit 2) shows an increase in the supply of 
TABLE 6

$\mathrm{P}_{2} \mathrm{O}_{5}$ Content in Sediments from Site 348 DSDP Leg 38

\begin{tabular}{|c|c|c|c|}
\hline Core & Section & $\begin{array}{l}\text { Interval } \\
(\mathrm{cm})\end{array}$ & $\mathrm{P}_{2} \mathrm{O}_{5}(\%)$ \\
\hline 1 & 3 & $100-104$ & 0.24 \\
\hline 1 & 3 & $100-104$ & 0.23 \\
\hline 1 & 5 & $90-92$ & 0.12 \\
\hline 1 & $\mathrm{CC}$ & & 0.17 \\
\hline 2 & 1 & $98-100$ & 0.18 \\
\hline 2 & 2 & $50-52$ & 0.22 \\
\hline 2 & $\mathrm{CC}$ & & 0.18 \\
\hline 3 & 1 & $99-101$ & 0.16 \\
\hline 3 & 1 & 99-101 & 0.18 \\
\hline 3 & 2 & $75-77$ & 0.08 \\
\hline 3 & 4 & 99-101 & 0.16 \\
\hline 3 & $\mathrm{CC}$ & & 0.11 \\
\hline 3 & $\mathrm{CC}$ & & 0.13 \\
\hline 4 & 1 & $119-121$ & 0.16 \\
\hline 5 & 1 & $99-100$ & 0.12 \\
\hline 5 & 2 & $134-141$ & 0.10 \\
\hline 6 & 5 & $73-75$ & 0.20 \\
\hline 6 & $\mathrm{CC}$ & & 0.19 \\
\hline 6 & $\mathrm{CC}$ & & 0.18 \\
\hline 7 & $\mathrm{CC}$ & & 0.13 \\
\hline 8 & 3 & $75-77$ & 0.11 \\
\hline 9 & 1 & $138-140$ & 0.10 \\
\hline 11 & 1 & $80-86$ & 0.15 \\
\hline 12 & 2 & $60-62$ & 0.17 \\
\hline 13 & $\mathrm{CC}$ & & 0.13 \\
\hline 14 & 2 & $20-22$ & 0.14 \\
\hline 14 & 6 & $20-22$ & 0.13 \\
\hline 14 & $\mathrm{CC}$ & & 0.12 \\
\hline 14 & $\mathrm{CC}$ & & 0.11 \\
\hline 15 & 1 & $60-62$ & 0.14 \\
\hline 15 & 2 & $60-62$ & 0.18 \\
\hline 15 & $\mathrm{CC}$ & & 0.08 \\
\hline 16 & 1 & $60-62$ & 0.14 \\
\hline 16 & 6 & $60-62$ & 0.13 \\
\hline 16 & 6 & $60-62$ & 0.10 \\
\hline 19 & 1 & $110-112$ & 0.11 \\
\hline 19 & 3 & $75-77$ & 0.09 \\
\hline 19 & 4 & $10-12$ & 0.08 \\
\hline 19 & 6 & $140-142$ & 0.38 \\
\hline 19 & $\mathrm{CC}$ & & 0.05 \\
\hline 20 & 1 & $80-82$ & 0.14 \\
\hline 20 & 1 & $80-82$ & 0.11 \\
\hline 20 & 4 & $64-66$ & 0.09 \\
\hline 20 & 6 & $135-137$ & 0.24 \\
\hline 20 & $\mathrm{CC}$ & & 0.16 \\
\hline 21 & 1 & $60-62$ & 0.09 \\
\hline 21 & 3 & $108-109$ & 0.08 \\
\hline 21 & $\mathrm{CC}$ & & 0.03 \\
\hline 22 & CC & & 0.27 \\
\hline 23 & 1 & $110-112$ & 0.22 \\
\hline 23 & $\mathrm{CC}$ & & 0.13 \\
\hline 23 & $\mathrm{CC}$ & & 0.13 \\
\hline 24 & $\mathrm{CC}$ & & 0.09 \\
\hline 25 & 4 & $75-77$ & 0.10 \\
\hline 25 & 6 & $71-73$ & 0.11 \\
\hline 25 & 6 & $71-73$ & 0.10 \\
\hline 25 & $\mathrm{CC}$ & & 0.10 \\
\hline 26 & 1 & $58-60$ & 0.16 \\
\hline 26 & 3 & $68-70$ & 0.13 \\
\hline 26 & $\mathrm{CC}$ & & 0.14 \\
\hline 27 & 1 & $92-96$ & 0.14 \\
\hline 27 & 2 & $103-105$ & 0.20 \\
\hline 28 & 1 & $75-77$ & 0.13 \\
\hline 28 & $\mathrm{CC}$ & & 0.18 \\
\hline 29 & 3 & $25-27$ & 0.11 \\
\hline 29 & $\mathrm{CC}$ & & 0.09 \\
\hline 30 & 1 & $76-78$ & 0.16 \\
\hline
\end{tabular}

TABLE 6 - Continued

\begin{tabular}{|c|c|c|c|}
\hline Core & Section & $\begin{array}{c}\text { Interval } \\
(\mathrm{cm})\end{array}$ & $\mathrm{P}_{2} \mathrm{O}_{5}(\%)$ \\
\hline 30 & 2 & $25-27$ & 0.12 \\
\hline 30 & 3 & $62-64$ & 0.10 \\
\hline 30 & 5 & $52-54$ & 0.14 \\
\hline 30 & 6 & $18-20$ & 0.12 \\
\hline 31 & 1 & $71-73$ & 0.10 \\
\hline
\end{tabular}

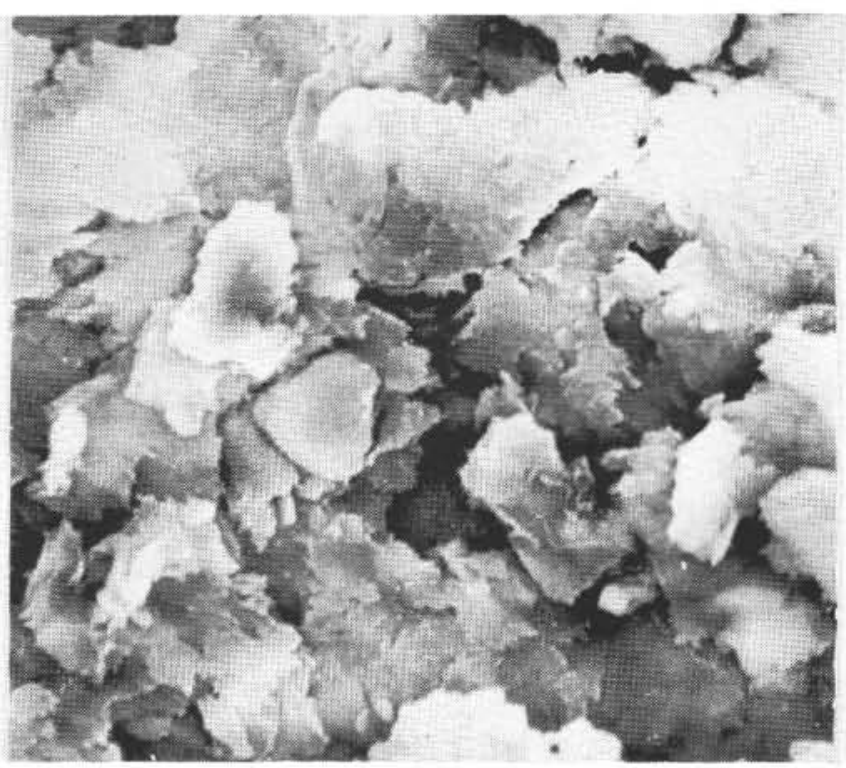

Figure 3. Less than $1 \mu \mathrm{m}$ fraction of sediments from a depth of 400-401.5 meters. Core 26. X4900 magnification.

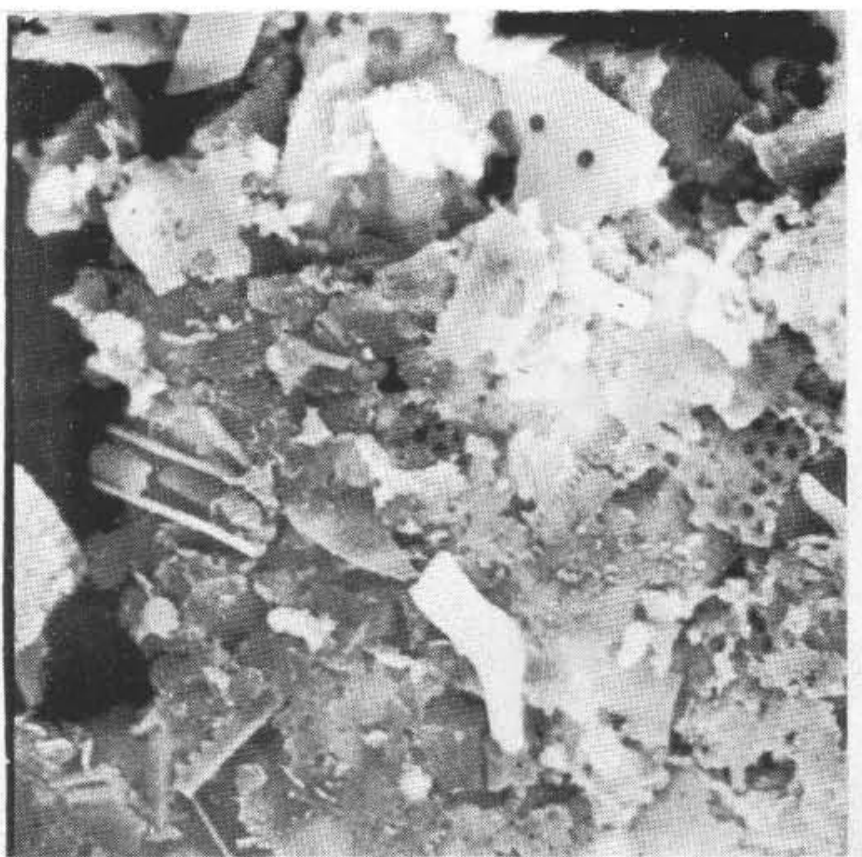

Figure 4. Less than $1 \mu \mathrm{m}$ fraction of sediments from a depth of 160.5-161 meters. Core 11, CC. X4900 magnification. 
TABLE 7

$\mathrm{SiO}_{2}$ Content from Soda Extraction in Sediments at Site 348 DSDP Leg 38

\begin{tabular}{|c|c|c|c|}
\hline Core & Section & $\begin{array}{c}\text { Interval } \\
(\mathrm{cm})\end{array}$ & $\mathrm{SiO}_{2}(\%)$ \\
\hline 1 & 3 & $100-101$ & 1.10 \\
\hline 1 & $\mathrm{CC}$ & & 1.44 \\
\hline 2 & 1 & $98-100$ & 1.20 \\
\hline 3 & 1 & $99-101$ & 1.28 \\
\hline 3 & $\mathrm{CC}$ & & 1.24 \\
\hline 5 & 2 & $139-141$ & 1.70 \\
\hline 6 & $\mathrm{CC}$ & & 4.56 \\
\hline 7 & 3 & $74-76$ & 5.00 \\
\hline 7 & 4 & $75-77$ & 2.56 \\
\hline 7 & $\mathrm{CC}$ & & 3.26 \\
\hline 8 & 2 & $75-77$ & 3.28 \\
\hline 8 & 3 & $75-77$ & 3.50 \\
\hline 9 & $\mathrm{CC}$ & & 3.50 \\
\hline 11 & 4 & $70-72$ & 7.04 \\
\hline 13 & 1 & $80-82$ & 6.14 \\
\hline 13 & $\mathrm{CC}$ & & 5.26 \\
\hline 14 & 1 & $20-22$ & 7.86 \\
\hline 14 & 2 & $20-22$ & 7.76 \\
\hline 14 & 4 & $20-22$ & 6.00 \\
\hline 14 & 5 & $20-22$ & 5.94 \\
\hline 14 & 6 & $20-22$ & 7.26 \\
\hline 14 & $\mathrm{CC}$ & & 5.30 \\
\hline 15 & 1 & $60-62$ & 8.14 \\
\hline 15 & 2 & $60-62$ & 7.84 \\
\hline 16 & 1 & $60-62$ & 6.30 \\
\hline 16 & 3 & $60-62$ & 8.04 \\
\hline 16 & 5 & $60-62$ & 8.60 \\
\hline 16 & 6 & $60-62$ & 8.06 \\
\hline 16 & $\mathrm{CC}$ & & 2.02 \\
\hline 19 & 4 & $10-12$ & 2.20 \\
\hline 19 & 6 & $140-142$ & 1.30 \\
\hline 19 & $\mathrm{CC}$ & & 0.76 \\
\hline 20 & 1 & $80-82$ & 1.56 \\
\hline 20 & 2 & $80-82$ & 1.35 \\
\hline 20 & 4 & $64-66$ & 1.98 \\
\hline 20 & 6 & $135-137$ & 1.30 \\
\hline 20 & $\mathrm{CC}$ & & 1.20 \\
\hline 21 & 1 & $60-62$ & 1.66 \\
\hline 21 & 3 & $100-104$ & 1.72 \\
\hline 21 & 5 & $60-62$ & 1.70 \\
\hline 21 & $\mathrm{CC}$ & & 1.28 \\
\hline 22 & $\mathrm{CC}$ & & 1.20 \\
\hline 23 & 1 & $110-112$ & 2.68 \\
\hline 23 & 3 & $77-79$ & 1.36 \\
\hline 23 & $\mathrm{CC}$ & & 1.46 \\
\hline 24 & $\mathrm{CC}$ & & 1.18 \\
\hline 25 & 4 & $75-77$ & 1.44 \\
\hline 25 & 6 & $71-73$ & 1.18 \\
\hline 25 & $\mathrm{CC}$ & & 0.70 \\
\hline 26 & 1 & $58-60$ & 1.32 \\
\hline 26 & 3 & $68-70$ & 1.10 \\
\hline 26 & $\mathrm{CC}$ & & 1.10 \\
\hline 27 & 1 & $92-96$ & 1.36 \\
\hline 27 & 2 & $103-105$ & 1.50 \\
\hline 28 & 1 & $75-77$ & 1.42 \\
\hline 28 & $\mathrm{CC}$ & & 0.86 \\
\hline 29 & 3 & $25-27$ & 1.34 \\
\hline 29 & $\mathrm{CC}$ & & 2.00 \\
\hline 30 & 1 & $96-98$ & 1.14 \\
\hline 30 & 2 & $25-29$ & 1.28 \\
\hline 30 & 3 & $62-64$ & 1.24 \\
\hline 30 & 5 & $52-54$ & 1.34 \\
\hline 30 & 6 & $18-20$ & 1.32 \\
\hline 31 & 1 & $71-73$ & 1.32 \\
\hline
\end{tabular}

ash. A substantially high amount of this material is present in the lower portions of Unit 2, particularly at the boundary with the early Miocene. The highest contents
TABLE 8

$\mathrm{CO}_{2}$ and $\mathrm{C}$ Organic Content (by Knopp's Method) in Sediments at Hole 348 DSDP Leg 38

\begin{tabular}{|c|c|c|c|c|}
\hline \multirow[b]{2}{*}{ Core } & \multirow[b]{2}{*}{ Section } & \multirow{2}{*}{$\begin{array}{c}\text { Interval } \\
(\mathrm{cm})\end{array}$} & \multicolumn{2}{|c|}{$\begin{array}{l}\text { Contents in } \\
\text { Dry Sample }\end{array}$} \\
\hline & & & $\mathrm{CO}_{2}$ & $\mathrm{C}_{\text {org }}$ \\
\hline 1 & 3 & $100-104$ & 1.04 & 0.27 \\
\hline 1 & $\mathrm{CC}$ & & 2.86 & 0.31 \\
\hline 3 & $\mathrm{CC}$ & & 0.16 & 0.32 \\
\hline 6 & $\mathrm{CC}$ & & 4.20 & 0.33 \\
\hline 7 & $\mathrm{CC}$ & & 0.05 & 0.36 \\
\hline 9 & $\mathrm{CC}$ & & 1.88 & 0.23 \\
\hline 13 & $\mathrm{CC}$ & & 0.10 & 0.10 \\
\hline 13 & $\mathrm{CC}$ & & 0.11 & 0.11 \\
\hline 14 & $\mathrm{CC}$ & & 0.06 & 0.31 \\
\hline 16 & 1 & $67-69$ & 0.13 & 1.47 \\
\hline 16 & $\mathrm{CC}$ & & 0.10 & 0.57 \\
\hline 19 & $\mathrm{CC}$ & & 0.23 & 0.11 \\
\hline 20 & $\mathrm{CC}$ & & 1.25 & 0.10 \\
\hline 21 & $\mathrm{CC}$ & & 0.13 & 0.22 \\
\hline 22 & $\mathrm{CC}$ & & 5.80 & 0.05 \\
\hline 23 & $\mathrm{CC}$ & & 0.14 & 0.42 \\
\hline 24 & $\mathrm{CC}$ & & 0.06 & 0.65 \\
\hline 25 & $\mathrm{CC}$ & & 0.05 & 0.84 \\
\hline 26 & $\mathrm{CC}$ & & 0.18 & 0.95 \\
\hline 28 & $\mathrm{CC}$ & & 0.13 & 0.91 \\
\hline 29 & $\mathrm{CC}$ & & 4.44 & 0.62 \\
\hline
\end{tabular}

of montmorillonite in the clay and zeolite in the silt fraction are present in the lower portions of the middle Miocene. Thus, the early/middle Miocene in the Icelandic Basin was a time of accumulation of essentially volcanogenic sediments.

Upward within Unit 2, the volcanogenic constituents decrease, giving way to an increase in terrigenous components. This is especially noticeable in the composition of the clay fraction where hydromicas and montmorillonites are sediment components. These changes are clearly seen in sediments of Unit 2, of late Pliocene age. This may indicate active glaciation with ice rafting being the main source of terrigenous material by late Pliocene times.

A distinctive feature of the volcanogenic sediment of Unit 2, when compared with the sediment of Unit 3, is the high degree of preservation of silt-sized ash material. A considerable portion of the glass is not yet devitrified. They may be explained by the relative young age of the sediments and the low content of organic matter. This, together with a sharp decrease of iron sulfidization, may indicate that in the middle, especially, upper Miocene a reconstruction of the water mass characteristics was occurring in the Icelandic Basin. This reconstruction may be a result of an increased influence of North Atlantic waters in the Norwegian-Greenland Sea due to a subsidence of barriers (Iceland-Faeroe Ridge).

Possibly, during the accumulation of Unit 2 there was a change in volcanic activity. This seems indicated by an increase of acid products upwards in Unit 2, with a maximum supply in the Pliocene.

Sedimentation in the Icelandic Basin during the middle-upper Miocene and Pliocene was taking place under climatic conditions that differed from those during the deposition of Units 3 and 1. In all probability, the climate was "mild" which resulted in a minimal effect by glaciation and/or ice-rafting. The temperature regime of the water masses became favorable, which, in 
TABLE 9

$\mathrm{Ca}$ and $\mathrm{Mg}$ Content in Carbonates in Sediments at Site 348 DSDP Leg 38

\begin{tabular}{|c|c|c|c|c|}
\hline Core & Section & $\begin{array}{l}\text { Interval } \\
(\mathrm{cm})\end{array}$ & $\mathrm{CaO}(\%)$ & $\mathrm{MgO}(\%)$ \\
\hline 1 & 3 & $100-104$ & 2.00 & 1.47 \\
\hline 1 & $\mathrm{CC}$ & & 4.34 & 1.41 \\
\hline 2 & 1 & $98-100$ & 8.34 & 1.48 \\
\hline 3 & 1 & 99-101 & 0.72 & 1.37 \\
\hline 3 & $\mathrm{CC}$ & & 0.72 & 1.04 \\
\hline 5 & 2 & $134-142$ & 1.42 & 2.32 \\
\hline 6 & $\mathrm{CC}$ & & 7.20 & 1.37 \\
\hline 7 & 3 & $74-76$ & 2.58 & 1.19 \\
\hline 7 & 4 & $75-77$ & 1.68 & 1.12 \\
\hline 7 & $\mathrm{CC}$ & & 1.08 & 1.02 \\
\hline 8 & 2 & $75-77$ & 1.08 & 0.89 \\
\hline 8 & 3 & $75-77$ & 1.52 & 1.12 \\
\hline 11 & 4 & $70-72$ & 2.18 & 1.21 \\
\hline 13 & 1 & $80-82$ & 1.18 & 0.75 \\
\hline 13 & $\mathrm{CC}$ & & 1.72 & 1.19 \\
\hline 14 & 1 & $20-22$ & 1.84 & 1.15 \\
\hline 14 & 2 & $20-22$ & 1.92 & 1.02 \\
\hline 14 & 4 & $20-22$ & 1.82 & 0.98 \\
\hline 14 & 6 & $20-22$ & 2.24 & 1.21 \\
\hline 14 & $\mathrm{CC}$ & & 1.96 & 1.06 \\
\hline 15 & 1 & $60-62$ & 1.92 & 0.95 \\
\hline 15 & 2 & $60-62$ & 2.28 & 1.05 \\
\hline 16 & 1 & $60-62$ & 1.92 & 1.09 \\
\hline 16 & 3 & $60-62$ & 1.24 & 0.78 \\
\hline 16 & 5 & $60-62$ & 1.96 & 0.88 \\
\hline 16 & 6 & $60-62$ & 2.24 & 0.96 \\
\hline 16 & $\mathrm{CC}$ & & 1.56 & 1.28 \\
\hline 19 & 4 & $10-12$ & 1.76 & 3.15 \\
\hline 19 & 6 & $140-142$ & 7.10 & 6.03 \\
\hline 19 & $\mathrm{CC}$ & & 1.64 & 6.02 \\
\hline 20 & 1 & $80-82$ & 3.24 & 5.41 \\
\hline 20 & 2 & $80-82$ & 2.00 & 4.92 \\
\hline 20 & 4 & $64-66$ & 1.08 & 1.28 \\
\hline 20 & 6 & $135-137$ & 17.16 & 4.18 \\
\hline 20 & $\mathrm{CC}$ & & 3.20 & 5.33 \\
\hline 21 & 1 & $60-62$ & 1.88 & 3.40 \\
\hline 21 & 3 & $100-104$ & 1.36 & 1.28 \\
\hline 21 & 5 & $60-62$ & 2.20 & 1.44 \\
\hline 21 & $\mathrm{CC}$ & & 1.80 & 4.87 \\
\hline 22 & $\mathrm{CC}$ & & 8.76 & 2.86 \\
\hline 23 & 1 & $110-112$ & 0.74 & 0.45 \\
\hline 23 & 3 & $77-79$ & 1.36 & 0.95 \\
\hline 23 & $\mathrm{CC}$ & & 1.16 & 0.89 \\
\hline 24 & $\mathrm{CC}$ & & 1.28 & 1.27 \\
\hline 25 & 4 & $75-77$ & 1.02 & 0.83 \\
\hline 25 & 6 & $71-73$ & 1.40 & 0.96 \\
\hline 25 & $\mathrm{CC}$ & & 3.48 & 1.37 \\
\hline 26 & 1 & $58-60$ & 0.84 & 0.94 \\
\hline 26 & 3 & $68-70$ & 1.16 & 0.97 \\
\hline 26 & $\mathrm{CC}$ & & 1.52 & 0.98 \\
\hline 27 & 1 & $92-96$ & 1.14 & 0.79 \\
\hline 27 & 2 & $103-105$ & 1.12 & 0.81 \\
\hline 28 & 2 & $75-77$ & 0.88 & 0.96 \\
\hline 28 & $\mathrm{CC}$ & & 1.36 & 0.75 \\
\hline 29 & 3 & $25-27$ & 0.96 & 0.78 \\
\hline 29 & $\mathrm{CC}$ & & 7.48 & 0.81 \\
\hline 30 & 1 & $76-78$ & 1.02 & 0.80 \\
\hline 30 & 2 & $25-27$ & 0.78 & 0.61 \\
\hline 30 & 3 & $62-64$ & 0.94 & 0.76 \\
\hline 30 & 5 & $52-54$ & 1.04 & 0.97 \\
\hline 30 & 6 & $18-20$ & 1.06 & 0.89 \\
\hline 31 & 1 & $71-73$ & 1.04 & 0.70 \\
\hline
\end{tabular}

combination with an active circulation, led to increased productivity. This is reflected by the increase of biogenic components in the sediments of Unit 2. The predominance of diatoms indicates the increase of temperature was not sufficient to exclude this area from the subtropical climatic zone.

The Pleistocene stage (Unit 1) of sedimentation in the Icelandic Basin is characterized by a sharp increase in the supply of terrigenous material. This material became the predominant sediment component in the Unit 1 sediments. Thus, the sediments can be considered to be volcanogenic-terrigenous.

The increase in the terrigenous components is associated with increased glaciation resulting from Pleistocene on Greenland and the Scandinavian Peninsula. The transport of ice-rafted terrigenous material had already been recorded in the upper Pliocene (Laughton, Berggren, et al., 1972). This cooling was responsible for a decrease in water mass temperatures in the North Atlantic, for a change of climatic conditions to "Arctic" types for the Norwegian-Greenland Sea, and for a certain reduction of primary productivity. The Pleistocene was notable only for rare local increases in productivity, expressed by a sharp increase of biogenic calcium carbonate and foraminiferal fragments in separate interlayers. Most of these maxima are most likely confined to the Holocene.

Since the change of the composition of the Pleistocene sediments was brought about by the increase in the supply of terrigenous material, it is difficult to establish whether there was a change in the rates of supply of volcanogenic material to the area. A decrease in the percentage of volcanogenic components in the Pleistocene sediments is caused primarily from dilution by terrigenous material. However, some indirect data indicate that during the Pleistocene, there was a decrease in the rate of supply of volcanogenic (mainly ash) material. This is testified by a sharp drop of the percentage of (fresh) volcanic glass in the silty fractions of these sediments.

Devitrificated glass (its quantity remains always high) is not a sufficiently exact indicator, inasmuch as part of this glass, in the form of terrigenous material, is supplied from the continental extrusive sources.

\section{SUMMARY}

1. Dominant volcanogenic sources were active during almost the entire period of late Oligocene, Miocene, and Pliocene. The main component is explosive material of a basic composition. The maximum influx corresponds to the boundary between early and middle Miocene. At the base of this primarily volcanogenic sequence, a basal sediment sequence exists whose composition indicates derivation by weathering of underlying basalts.

2 . The major portion of the volcanogenic material has been subjected to reworking and alteration to montmorillonite clay. These changes are most vividly manifested in older late Oligocene and early Miocene sediments.

3. Pleistocene sediments represent mixed volcanogenic-terrigenous sources. This can be explained by climatic change (cooling) and by a decrease of volcanic activity.

4. Biogenic component (silica, calcium carbonate) in the late Cenozoic sediments is subordinate. The maximum accumulation was during the middle and. late Miocene and in early Pliocene. 


\section{ACKNOWLEDGMENTS}

All analyses on the sediments from Site 348 were performed at the Analytical Laboratory of the P.P. Shirshov Institute of Oceanology, Academy of Sciences, USSR. The analyses were performed by A.G. Samosudova, N.P. Tolmacheva, M.B. Chermashenzeva, N.W. Turanskaya, T.G. Kuzmina, L.I. Streljanova, S.N. Koptilkina, W.P. Kasakova, A.N. Rudakova, A.Ja. Shevchenko, E.A. Marketov, W.A. Karlov, and O.S. Dimitrenko.

\section{REFERENCES}

Brindley, G. V., 1965. Quantitative analysis of mixtures of clay minerals: Bull. X-Ray Methods for Study of Clay Minerals: Moscow (Mir Publishers).

Laughton, A. S., Berggren, W. A., et al., 1972. Initial Reports of the Deep Sea Drilling Project, Volume 12: Washington (U.S. Government Printing Office), p. 1-1243. 